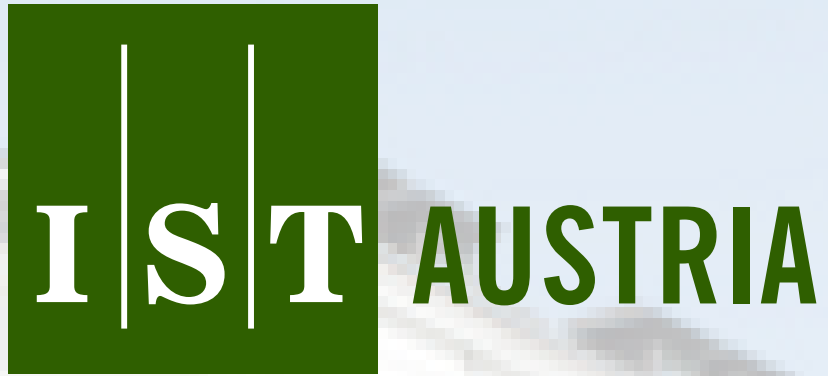

Institute of Science and Technology

\title{
Faster Algorithms for Quantitative Verification in Constant
} Treewidth Graphs

Krishnendu Chatterjee and Rasmus Ibsen-Jensen and Andreas Pavlogiannis

Technical Report No. IST-2015-319-v1+1

Deposited at 10 Feb 2015 08:58

http://repository.ist.ac.at/319/1/long.pdf

IST Austria (Institute of Science and Technology Austria)

Am Campus 1

A-3400 Klosterneuburg, Austria 
Copyright (C) 2012, by the author(s).

All rights reserved.

Permission to make digital or hard copies of all or part of this work for personal or classroom use is granted without fee provided that copies are not made or distributed for profit or commercial advantage and that copies bear this notice and the full citation on the first page. To copy otherwise, to republish, to post on servers or to redistribute to lists, requires prior specific permission. 


\title{
Faster Algorithms for Quantitative Verification in Constant Treewidth Graphs
}

\author{
Krishnendu Chatterjee $^{\dagger} \quad$ Rasmus Ibsen-Jensen $^{\dagger} \quad$ Andreas Pavlogiannis $^{\dagger}$ \\ ${ }^{\dagger}$ IST Austria
}

\begin{abstract}
We consider the core algorithmic problems related to verification of systems with respect to three classical quantitative properties, namely, the meanpayoff property, the ratio property, and the minimum initial credit for energy property. The algorithmic problem given a graph and a quantitative property asks to compute the optimal value (the infimum value over all traces) from every node of the graph. We consider graphs with constant treewidth, and it is well-known that the control-flow graphs of most programs have constant treewidth. Let $n$ denote the number of nodes of a graph, $m$ the number of edges (for constant treewidth graphs $m=O(n)$ ) and $W$ the largest absolute value of the weights. Our main theoretical results are as follows. First, for constant treewidth graphs we present an algorithm that approximates the mean-payoff value within a multiplicative factor of $\epsilon$ in time $O(n \cdot \log (n / \epsilon))$ and linear space, as compared to the classical algorithms that require quadratic time. Second, for the ratio property we present an algorithm that for constant treewidth graphs works in time $O(n \cdot \log (|a \cdot b \cdot n|))=O(n \cdot \log (n \cdot W))$, when the output is $\frac{a}{b}$, as compared to the previously best known algorithm with running time $O\left(n^{2} \cdot \log (n \cdot W)\right)$. Third, for the minimum initial credit problem we show that (i) for general graphs the problem can be solved in $O\left(n^{2} \cdot m\right)$ time and the associated decision problem can be solved in $O(n \cdot m)$ time, improving the previous known $O\left(n^{3} \cdot m \cdot \log (n \cdot W)\right)$ and $O\left(n^{2} \cdot m\right)$ bounds, respectively; and (ii) for constant treewidth graphs we present an algorithm that requires $O(n \cdot \log n)$ time, improving the previous known $O\left(n^{4} \cdot \log (n \cdot W)\right)$ bound. We have implemented some of our algorithms and show that they present a significant speedup on standard benchmarks.
\end{abstract}

\section{Introduction}

Boolean vs quantitative verification. The traditional view of verification has been qualitative (Boolean) that classifies traces of a system as "correct" vs "incorrect". In the recent years, motivated by applications to analyze resource-constrained systems (such as embedded systems), there has been a huge interest to study quantitative properties of systems. A quantitative property assigns to each trace of a system a real-number that quantifies how good or bad the trace is, instead of classifying it as correct vs incorrect. For example, a Boolean property may require that every request is eventually granted, whereas a quantitative property for each trace can measure the average waiting time between requests and corresponding grants.

Variety of results. Given the importance of quantitative verification, the traditional qualitative view of verification has been extended in several ways, such as, quantitative languages and quantitative automata for specification languages [27|17|16|21|15 477|28]; quantitative logics for specification languages [8|10|2]; quantitative synthesis for robust reactive systems [4|5|20]; a framework for quantitative 
abstraction refinement [13]; quantitative analysis of infinite-state systems [22]18]; and model measuring (that extends model checking) [33], to name a few. The core algorithmic question for many of the above studies is a graph algorithmic problem that requires to analyze a graph wrt a quantitative property.

Important quantitative properties. The three quantitative properties that have been studied for their relevance in analysis of reactive systems are as follows. First, the meanpayoff property consists of a weight function that assigns to every transition an integervalued weight and assigns to each trace the long-run average of the weights of the transitions of the trace. Second, the ratio property consists of two weight functions (one of which is a positive weight function) and assigns to each trace the ratio of the two mean-payoff properties (the denominator is wrt the positive function). The minimum initial credit for energy property consists of a weight function (like in the mean-payoff property) and assigns to each trace the minimum number to be added such that the partial sum of the weights for every prefix of the trace is non-negative. For example, the mean-payoff property is used for average waiting time, worst-case execution time analysis [17|13 |18]; the ratio property is used in robustness analysis of systems [5]; and the minimum initial credit for energy for measuring resource consumptions [9].

Algorithmic problems. Given a graph and a quantitative property, the value of a node is the infimum value of all traces that start at the respective node. The algorithmic problem (namely, the value problem) for analysis of quantitative properties consists of a graph and a quantitative property, and asks to compute either the exact value or an approximation of the value for every node in the graph. The algorithmic problems are at the heart of many applications, such as automata emptiness, model measuring, quantitative abstraction refinement, etc.

Treewidth of graphs. A very well-known concept in graph theory is the notion of treewidth of a graph, which is a measure of how similar a graph is to a tree (a graph has treewidth 1 precisely if it is a tree) [43]. The treewidth of a graph is defined based on a tree decomposition of the graph [31], see Section2 for a formal definition. Beyond the mathematical elegance of the treewidth property for graphs, there are many classes of graphs which arise in practice and have constant treewidth. The most important example is that the control flow graphs of goto-free programs for many programming languages are of constant treewidth [45], and it was also shown in [30] that typically all Java programs have constant treewidth. For many other applications see the surveys [677]. The constant treewidth property of graphs has also played an important role in logic and verification; for example, MSO (Monadic Second Order logic) queries can be solved in polynomial time [24] (also in log-space [29]) for constant-treewidth graphs; parity games on graphs with constant treewidth can be solved in polynomial time [39]; and there exist faster algorithms for probabilistic models (like Markov decision processes) [14]. Moreover, recently it has been shown that the constant treewidth property is also useful for interprocedural analysis [18].

Previous results and our contributions. In this work we consider general graphs and graphs with constant treewidth, and the algorithmic problems to compute the exact value or an approximation of the value for every node wrt to quantitative properties given as the mean-payoff, the ratio, or the minimum initial credit for energy. We first present the relevant previous results, and then our contributions. 


\begin{tabular}{|c|c|c|c|c|c|}
\hline \multicolumn{4}{|c|}{ Minimum mean-cycle value } & \multicolumn{3}{c|}{ Minimum ratio-cycle value } \\
\hline \hline Orlin \& Ahuja [40] & Karp [34] & $\begin{array}{c}\text { Our result [Thm[4] } \\
(\epsilon \text {-approximate) }\end{array}$ & Burns [12] & Lawler [37] & Our result [Cor 2] \\
\hline$O\left(n^{1.5} \cdot \log (n \cdot W)\right)$ & $O\left(n^{2}\right)$ & $\mathbf{O}(\mathbf{n} \cdot \log (\mathbf{n} / \epsilon))$ & $O\left(n^{3}\right)$ & $O\left(n^{2} \cdot \log (n \cdot W)\right)$ & $\mathbf{O}(\mathbf{n} \cdot \log (|\mathbf{a} \cdot \mathbf{b} \cdot \mathbf{n}|))$ \\
\hline
\end{tabular}

Table 1: Time complexity of existing and our solutions for the minimum mean-cycle value and ratio-cycle value problem in constant treewidth weighted graphs with $n$ nodes and largest absolute weight $W$, when the output is the (irreducible) fraction $\frac{a}{b}$.

\begin{tabular}{|c|c|c|c|}
\hline & Bouyer et. al. [9] & $\begin{array}{c}\text { Our result } \\
{[\mathbf{T h m} \text { 5. Cor 3 }}\end{array}$ & $\begin{array}{c}\text { Our result [Thm]7] } \\
\text { (constant treewidth) }\end{array}$ \\
\hline \hline Time (decision) & $O\left(n^{2} \cdot m\right)$ & $\mathbf{O}(\mathbf{n} \cdot \mathbf{m})$ & $\mathbf{O}(\mathbf{n} \cdot \log \mathbf{n})$ \\
\hline Time & $O\left(n^{3} \cdot m \cdot \log (n \cdot W)\right)$ & $\mathbf{O}\left(\mathbf{n}^{2} \cdot \mathbf{m}\right)$ & $\mathbf{O}(\mathbf{n} \cdot \log \mathbf{n})$ \\
\hline Space & $O(n)$ & $\mathbf{O}(\mathbf{n})$ & $\mathbf{O}(\mathbf{n})$ \\
\hline
\end{tabular}

Table 2: Complexity of the existing and our solution for the minimum initial credit problem on weighted graphs of $n$ nodes, $m$ edges, and largest absolute weight $W$.

Previous results. We consider graphs with $n$ nodes, $m$ edges, and let $W$ denote the largest absolute value of the weights. The running time of the algorithms is characterized by the number of arithmetic operations (i.e., each operation takes constant time); and the space is characterized by the maximum number of integers the algorithm stores. The classical algorithm for graphs with mean-payoff properties is the minimum meancycle problem of Karp [34], and the algorithm requires $O(n \cdot m)$ running time and $O\left(n^{2}\right)$ space. A different algorithm was proposed in [38] that requires $O(n \cdot m)$ running time and $O(n)$ space. Orlin and Ahuja [40] gave an algorithm running in time $O(\sqrt{n} \cdot m \cdot \log (n \cdot W))$. For some special cases there exist faster approximation algorithms [19]. There is a straightforward reduction of the ratio problem to the mean-payoff problem. For computing the exact minimum ratio, the fastest known strongly polynomial time algorithm is Burns' algorithm [12] running in time $O\left(n^{2} \cdot m\right)$. Also, there is an algorithm by Lawler [37] that uses $O(n \cdot m \cdot \log (n \cdot W))$ time. Many pseudopolynomial algorithms are known for the problem, with polynomial dependency on the numbers appearing in the weight function, see [26]. For the minimum initial credit for energy problem, the decision problem (i.e., is the energy required for node $v$ at most $c$ ?) can be solved in $O\left(n^{2} \cdot m\right)$ time, leading to an $O\left(n^{3} \cdot m \cdot \log (n \cdot W)\right)$ time algorithm for the minimum initial credit for energy problem [9]. All the above algorithms are for general graphs (without the constant-treewidth restriction).

Our contributions. Our main contributions are as follows.

1. Finding the mean-payoff and ratio values in constant-treewidth graphs. We present two results for constant treewidth graphs. First, for the exact computation we present an algorithm that requires $O(n \cdot \log (|a \cdot b \cdot n|))$ time and $O(n)$ space, where $\frac{a}{b} \neq 0$ is the (irreducible) ratio/mean-payoff of the output. If $\frac{a}{b}=0$ then the algorithm uses $O(n \cdot \log n)$ time. Note that $\log (|a \cdot b \cdot n|) \leq 3 \log (n \cdot W)$. We also present a space-efficient version of the algorithm that requires only $O(\log n)$ space. Second, we present an algorithm for finding an $\epsilon$-factor approximation that requires $O(n \cdot \log (n / \epsilon))$ time and $O(n)$ space, as compared to the $O\left(n^{1.5} \cdot \log (n \cdot W)\right)$ time solution of Orlin \& Ahuja, and the $O\left(n^{2}\right)$ time solution of Karp (see Table 1).

2. Finding the minimum initial credit in graphs. We present two results. First, we consider the exact computation for general graphs, and present (i) an $O(n \cdot m)$ time algorithm for the decision problem (improving the previous known $O\left(n^{2}\right.$. 
$m$ ) bound), and (ii) an $O\left(n^{2} \cdot m\right)$ time algorithm to compute value of all nodes (improving the previous known $O\left(n^{3} \cdot m \cdot \log (n \cdot W)\right)$ bound). Finally, we consider the computation of the exact value for graphs with constant treewidth and present an algorithm that requires $O(n \cdot \log n)$ time (improving the previous known $O\left(n^{4}\right.$. $\log (n \cdot W)$ ) bound) (see Table 2,

3. Experimental results. We have implemented our algorithms for the minimum mean cycle and minimum initial credit problems and ran them on standard benchmarks (DaCapo suit [3] for the minimum mean cycle problem, and DIMACS challenges [1] for the minimum initial credit problem). For the minimum mean cycle problem, our results show that our algorithm has lower running time than all the classical polynomial-time algorithms. For the minimum initial credit problem, our algorithm provides a significant speedup over the existing method. Both improvements are demonstrated even on graphs of small/medium size. Note that our theoretical improvements (better asymptotic bounds) imply improvements for large graphs, and our improvements on medium size graphs indicate that our algorithms have practical applicability with small constants.

Technical contributions. The key technical contributions of our work are as follows:

1. Mean-payoff and ratio values in constant-treewidth graphs. Given a graph with constant treewidth, let $c^{*}$ be the smallest weight of a simple cycle. First, we present a linear-time algorithm that computes $c^{*}$ exactly (if $c^{*} \geq 0$ ) or approximate within a polynomial factor (if $c^{*}<0$ ). Then, we show that if the minimum ratio value $\nu^{*}$ is the irreducible fraction $\frac{a}{b}$, then $\nu^{*}$ can be computed by evaluating $O(\log (|a \cdot b \cdot n|))$ inequalities of the form $\nu^{*} \geq \nu$. Each such inequality is evaluated by computing the smallest weight of a simple cycle in a modified graph. Finally, for $\epsilon$-approximating the value $\nu^{*}$, we show that $O(\log (n / \epsilon))$ such inequalities suffice.

2. Minimum initial credit problem. We show that for general graphs, the decision problem can be solved with two applications of Bellman-Ford-type algorithms, and the value problem reduces to finding non-positive cycles in the graph, followed by one instance of the single-source shortest path problem. We then show how the invariants of the algorithm for the value problem on general graphs can be maintained by a particular graph traversal of the tree-decomposition for constant-treewidth graphs.

Our algorithms are simple and easily implementable.

\section{Definitions}

Weighted graphs. We consider finite weighted directed graphs $G=\left(V, E, \mathrm{wt}, \mathrm{wt}^{\prime}\right)$ where $V$ is the set of $n$ nodes, $E \subseteq V \times V$ is the edge relation of $m$ edges, wt $: E \rightarrow \mathbb{Z}$ is a weight function that assigns an integer weight $\mathrm{wt}(e)$ to each edge $e \in E$, and $\mathrm{wt}^{\prime}: E \rightarrow \mathbb{N}^{+}$is a weight function that assigns strictly positive integer weights. For technical simplicity, we assume that there exists at least one outgoing edge from every node. In certain cases where the function $\mathrm{wt}^{\prime}$ is irrelevant, we will consider weighted graphs $G=(V, E, \mathrm{wt})$, i.e., without the function $\mathrm{wt}^{\prime}$.

Finite and infinite paths. A finite path $P=\left(u_{1}, \ldots, u_{j}\right)$, is a sequence of nodes $u_{i} \in V$ such that for all $1 \leq i<j$ we have $\left(u_{i}, u_{i+1}\right) \in E$. The length of $P$ is $|P|=j-1$. A single-node path has length 0 . The path $P$ is simple if there is no node repeated in $P$, and it is a cycle if $j>1$ and $u_{1}=u_{j}$. The path $P$ is a simple cycle if $P$ is 
a cycle and the sequence $\left(u_{2}, \ldots u_{j}\right)$ is a simple path. The functions wt and wt' naturally extend to paths, so that the weight of a path $P$ with $|P|>0$ and wrt the weight functions wt and $\mathrm{wt}^{\prime}$ is $\mathrm{wt}(P)=\sum_{1 \leq i<j} \mathrm{wt}\left(u_{i}, u_{i+1}\right)$ and $\mathrm{wt}^{\prime}(P)=\sum_{1 \leq i<j} \mathrm{wt}^{\prime}\left(u_{i}, u_{i+1}\right)$. The value of $P$ is defined to be $\overline{\mathrm{wt}}(P)=\frac{\mathrm{wt}(P)}{\mathrm{wt}(P)}$. For the case where $|P|=0$, we define $\operatorname{wt}(P)=0$, and $\overline{\mathrm{wt}}(P)$ is undefined. An infinite path $\mathcal{P}=\left(u_{1}, u_{2}, \ldots\right)$ of $G$ is an infinite sequence of nodes such that every finite prefix $P$ of $\mathcal{P}$ is a finite path of $G$. The functions wt and $\mathrm{wt}^{\prime}$ assign to $\mathcal{P}$ a value in $\mathbb{Z} \cup\{-\infty, \infty\}$ : we have $\mathrm{wt}(\mathcal{P})=$ $\sum_{i} \operatorname{wt}\left(u_{i}, u_{i+1}\right)$ and $\mathrm{wt}^{\prime}(\mathcal{P})=\infty$. For a (possibly infinite) path $P$, we use the notation $u \in P$ to denote that a node $u$ appears in $P$, and $e \in P$ to denote that an edge $e$ appears in $P$. Given a set $B \subseteq V$, we denote with $P \cap B$ the set of nodes of $B$ that appear in $P$. Given a finite path $P_{1}$ and a possibly infinite path $P_{2}$, we denote with $P_{1} \circ P_{2}$ the path resulting from the concatenation of $P_{1}$ and $P_{2}$.

Distances and witness paths. For nodes $u, v \in V$, we denote with $d(u, v)=$ $\inf _{P: u \rightsquigarrow v} \operatorname{wt}(P)$ the distance from $u$ to $v$. A finite path $P: u \rightsquigarrow v$ is a witness of the distance $d(u, v)$ if $\operatorname{wt}(P)=d(u, v)$. An infinite path $\mathcal{P}$ is a witness of the distance $d(u, v)$ if the following conditions hold:

1. $d(u, v)=\operatorname{wt}(\mathcal{P})=-\infty$, and

2. $\mathcal{P}$ starts from $u$, and $v$ is reachable from every node of $\mathcal{P}$.

Note that $d(u, v)=\infty$ is not witnessed by any path.

Tree decompositions. A tree-decomposition $\operatorname{Tree}(G)=\left(V_{T}, E_{T}\right)$ of $G$ is a tree such that the following conditions hold:

1. $V_{T}=\left\{B_{0}, \ldots, B_{n^{\prime}-1}: \forall i B_{i} \subseteq V\right\}$ and $\bigcup_{B_{i} \in V_{T}} B_{i}=V$ (every node is covered).

2. For all $(u, v) \in E$ there exists $B_{i} \in V_{T}$ such that $u, v \in B_{i}$ (every edge is covered).

3. For all $i, j, k$ such that there is a bag $B_{k}$ that appears in the simple path $B_{i} \rightsquigarrow B_{j}$ in Tree $(G)$, we have $B_{i} \cap B_{j} \subseteq B_{k}$ (every node appears in a contiguous subtree of $\operatorname{Tree}(G))$.

The sets $B_{i}$ which are nodes in $V_{T}$ are called bags. Conventionally, we call $B_{0}$ the root of Tree $(G)$, and denote with $\operatorname{Lv}\left(B_{i}\right)$ the level of $B_{i}$ in Tree $(G)$, with $\operatorname{Lv}\left(B_{0}\right)=0$. We say that $\operatorname{Tree}(G)$ is balanced if the maximum level is $\max _{B_{i}} \operatorname{Lv}\left(B_{i}\right)=O\left(\log n^{\prime}\right)$, and it is binary if every bag has at most two children bags. A bag $B$ is called the root bag of a node $u$ if $B$ is the smallest-level bag that contains $u$, and we often use $B_{u}$ to refer to the root bag of $u$. The width of a tree-decomposition Tree $(G)$ is the size of the largest bag minus 1 . The treewidth of $G$ is the smallest width among the widths of all possible tree decompositions of $G$. The following lemma gives a fundamental structural property of tree-decompositions.

Lemma 1. Consider a graph $G=(V, E)$, a binary tree-decomposition $T=\operatorname{Tree}(G)$ and a bag $B$ of $T$. Denote with $\left(\mathcal{C}_{i}\right)_{1 \leq i \leq 3}$ the components of $T$ created by removing $B$ from $T$, and let $V_{i}$ be the set of nodes that appear in bags of component $\mathcal{C}_{i}$. For every $i \neq j$, nodes $u \in V_{i}, v \in V_{j}$ and $P: u \rightsquigarrow v$, we have that $P \cap B \neq \emptyset$ (i.e., all paths between $u$ and $v$ go through some node in $B$ ).

Theorem 1. For every graph $G$ with $n$ nodes and constant treewidth, a balanced binary tree-decomposition $\operatorname{Tree}(G)$ of constant width and $O(n)$ bags can be constructed in (1) $O(n \cdot \log n)$ time and $O(n)$ space [42], (2) deterministic logspace (and hence polynomial time) [29]. 
In the sequel we consider only balanced and binary tree-decompositions of constant width and $n^{\prime}=O(n)$ bags (and hence of height $O(\log n)$ ). Additionally, we consider that every bag is the root bag of at most one node. Obtaining this last property is straightforward, simply by replacing each bag $B$ which is the root of $k>1$ nodes $x_{1}, \ldots x_{k}$ with a chain of bags $B_{1}, \ldots, B_{k}=B$, where each $B_{i}$ is the parent of $B_{i+1}$, and $B_{i+1}=B_{i} \cup\left\{x_{i+1}\right\}$. Note that this keeps the tree binary and increases its height by at most a constant factor, hence the resulting tree is also balanced.

Throughout the paper, we follow the convention that the maximum and minimum of the empty set is $-\infty$ and $\infty$ respectively, i.e., $\max (\emptyset)=-\infty$ and $\min (\emptyset)=\infty$. Time complexity is measured in number of arithmetic and logical operations, and space complexity is measured in number of machine words. Given a graph $G$, we denote with $\mathcal{T}(G)$ and $\mathcal{S}(G)$ the time and space required for constructing a balanced, binary tree-decomposition Tree $(G)$. We are interested in the following problems.

The minimum mean cycle problem [35]. Given a weighted directed graph $G=$ $(V, E, \mathrm{wt})$, the minimum mean cycle problem asks to determine for each node $u$ the mean value $\mu^{*}(u)=\min _{C \in \mathcal{C}_{u}} \frac{\mathrm{wt}(C)}{|C|}$, where $\mathcal{C}_{u}$ is the set of simple cycles reachable from $u$ in $G$. Such a $C$ is called a minimum mean cycle of $u$. For $0<\epsilon<1$, we say that a value $\mu$ is an $\epsilon$-approximation of the mean value $\mu^{*}(u)$ if $\left|\mu-\mu^{*}(u)\right| \leq \epsilon \cdot\left|\mu^{*}(u)\right|$.

The minimum ratio cycle problem [32]. This is a generalization of the minimum mean cycle problem. Given a weighted directed graph $G=\left(V, E, w_{t}, w^{\prime}\right)$, the minimum ratio cycle problem asks to determine for each node $u$ the ratio value $\nu^{*}(u)=$ $\min _{C \in \mathcal{C}_{u}} \overline{\mathrm{wt}}(C)$, where $\overline{\mathrm{wt}}(C)=\frac{\mathrm{wt}(C)}{\mathrm{wt}^{\prime}(C)}$ and $\mathcal{C}_{u}$ is the set of simple cycles reachable from $u$ in $G$. Such a $C$ is called a minimum ratio cycle of $u$. The minimum mean cycle problem follows as a special case of the minimum ratio cycle problem for $\mathrm{wt}^{\prime}(e)=1$ for each edge $e \in E$.

The minimum initial credit problem [9]. Given a weighted directed graph $G=$ $(V, E, \mathrm{wt})$, the minimum initial credit value problem asks to determine for each node $u$ the smallest energy value $\mathrm{E}(u) \in \mathbb{N} \cup\{\infty\}$ with the following property: there exists an infinite path $\mathcal{P}=\left(u_{1}, u_{2} \ldots\right)$ with $u=u_{1}$, such that for every finite prefix $P$ of $\mathcal{P}$ we have $\mathrm{E}(u)+$ wt $(P) \geq 0$. Conventionally, we let $\mathrm{E}(u)=\infty$ if no finite value exists. The associated decision problem asks given a node $u$ and an initial credit $c \in \mathbb{N}$ whether $\mathrm{E}(u) \leq c$.

\section{Minimum Cycle}

In the current section we deal with a related graph problem, namely the detection of a minimum-weight simple cycle of a graph. In Section 4 we use solutions to the minimum cycle problem to obtain the minimum ratio and minimum mean values of a graph.

The minimum cycle problem. Given a weighted graph $G=(V, E$, wt $)$, the minimum cycle problem asks to determine the weight $c^{*}$ of a minimum-weight simple cycle in $G$, i.e., $c^{*}=\min _{C}$ wt $(C)$, where $C$ are taken to be simple cycles in $G$.

We describe the algorithm MinCycle that operates on a tree-decomposition $\operatorname{Tree}(G)$ of an input graph $G$, and has the following properties.

1. If $G$ has no negative cycles, then MinCycle returns the weight $c^{*}$ of a minimumweight cycle in $G$. 
2. If $G$ has negative cycles, then MinCycle returns a value that is at most a polynomial (in $n$ ) factor smaller than $c^{*}$.

U-shaped paths. Following the recent work of [18], we define the important notion of $\mathrm{U}$-shaped paths in a tree-decomposition Tree $(G)$. Given a bag $B$ and nodes $u, v \in B$, we say that a path $P: u \rightsquigarrow v$ is U-shaped in $B$, if one of the following conditions hold:

1. Either $|P|>1$ and for all intermediate nodes $w \in P$, we have $B$ is an ancestor of $B_{w}$,

2. or $|P| \leq 1$ and $B$ is $B_{u}$ or $B_{v}$ (i.e., $B$ is the root bag of either $u$ or $v$ ).

Informally, given a bag $B$, a $\mathrm{U}$-shaped path in $B$ is a path that traverses intermediate nodes that exist only in the subtree of $\operatorname{Tree}(G)$ rooted in $B$. The following remark follows from the definition of tree-decompositions, and states that every simple cycle $C$ can be seen as a U-shaped path $P$ from the smallest-level node of $C$ to itself. Consequently, we can determine the value $c^{*}$ by only considering U-shaped paths in $\operatorname{Tree}(G)$.

Remark 1. Let $C=\left(u_{1}, \ldots, u_{k}\right)$ be a simple cycle in $G$, and $u_{j}=$ $\arg \min _{u_{i} \in C} \operatorname{Lv}\left(u_{i}\right)$. Then $P=\left(u_{j}, u_{j+1}, \ldots u_{k}, u_{1}, \ldots, u_{j}\right)$ is a U-shaped path in $B_{u_{j}}$, and $\mathrm{wt}(P)=\mathrm{wt}(C)$.

Informal description of MinCycle. Based on U-shaped paths, the work of [18] presented a method for computing algebraic path properties on tree-decompositions with constant width, where the weights of the edges come from a general semiring. Note that integer-valued weights are a special case of the tropical semiring. Our algorithm MinCycle is similar to the algorithm Preprocess from [18]. It consists of a depth-first traversal of Tree $(G)$, and for each examined bag $B$ computes a local distance map $\mathrm{LD}_{B}: B \times B \rightarrow \mathbb{Z} \cup\{\infty\}$ such that for each $u, v \in B$, we have (i) $\operatorname{LD}_{B}(u, v)=\operatorname{wt}(P)$ for some path $P: u \rightsquigarrow v$, and (ii) $\mathrm{LD}_{B} \leq \min _{P}$ wt $(P)$, where $P$ are taken to be simple $u \rightsquigarrow v$ paths (or simple cycles) that are U-shaped in $B$. This is achieved by traversing Tree $(G)$ in post-order, and for each root bag $B_{x}$ of a node $x$, we update $\operatorname{LD}_{B_{x}}(u, v)$ with $\operatorname{LD}_{B_{x}}(u, x)+\operatorname{LD}_{B_{x}}(x, v)$ (i.e., we do path-shortening from node $u$ to node $v$, by considering paths that go through $x$ ). See Figure 1 for an illustration.

In the end, MinCycle returns $\min _{x} \mathrm{LD}_{B_{x}}(x, x)$, i.e., the weight of the smallest-weight $\mathrm{U}$-shaped (not necessarily simple) cycle it has discovered. Algorithm 1 gives MinCycle in pseudocode. For brevity, in line 5 we consider that if $\{u, v\} \notin E$ or $\{u, v\} \nsubseteq B_{i}$ for some child $B_{i}$ of $B$, then $\operatorname{LD}_{B_{i}}(u, v)=\infty$.

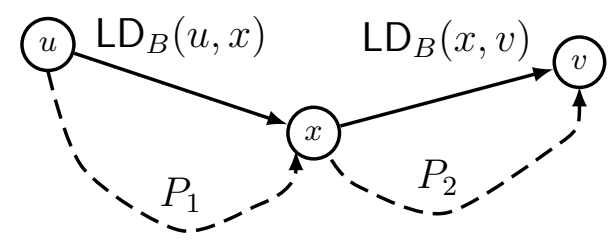

Fig. 1: Path shortening in line 10 of MinCycle. When $B_{x}$ is examined, $\operatorname{LD}_{B_{x}}(u, v)$ is updated with the weight of the $\mathrm{U}$-shaped path $P=P_{1} \circ P_{2}$. The paths $P_{1}$ and $P_{2}$ are $\mathrm{U}$-shaped paths in the children bags $B_{1}$ and $B_{2}$, and we have $\operatorname{LD}_{B_{i}}(u, x)=\operatorname{wt}\left(P_{i}\right)$. 


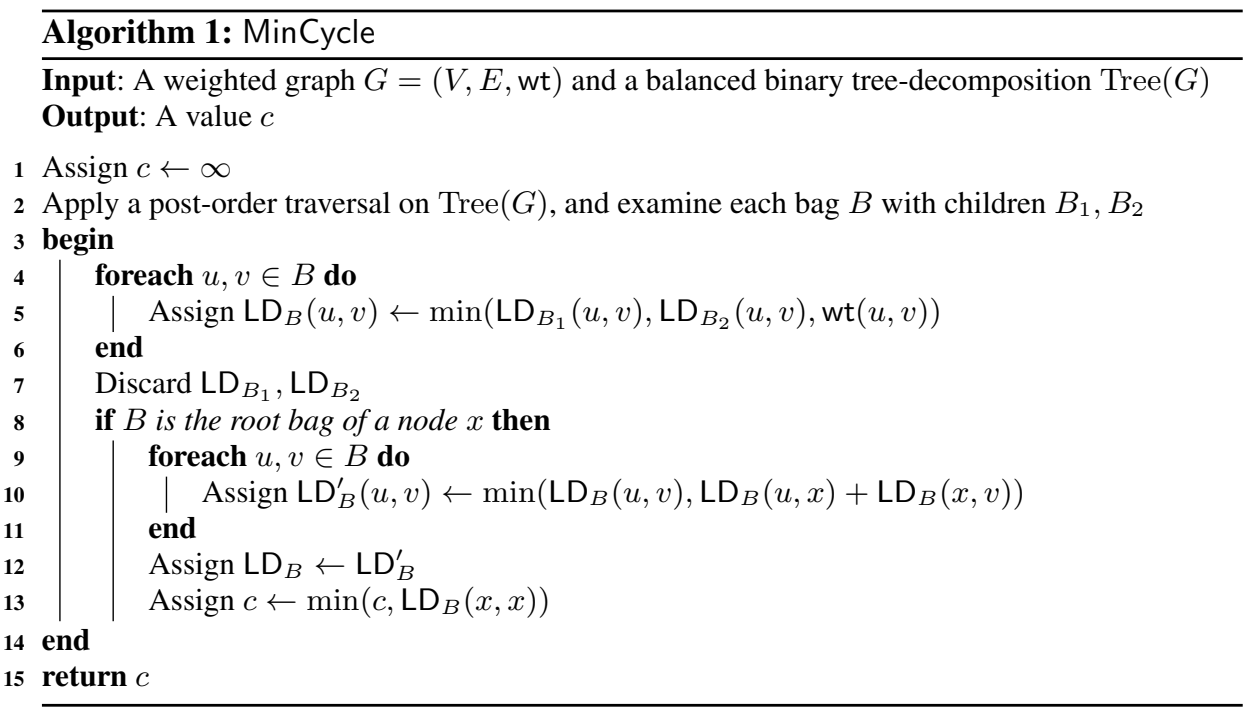

In essence, MinCycle performs repeated summarizations of paths in $G$. The following lemma follows easily from [18, Lemma 2], and states that $\operatorname{LD}_{B}(u, v)$ is upper bounded by the smallest weight of a U-shaped simple $u \rightsquigarrow v$ path in $B$.

Lemma 2 ([18, Lemma 2]). For every examined bag $B$ and nodes $u, v \in B$, we have

1. $\operatorname{LD}_{B}(u, v)=\operatorname{wt}(P)$ for some path $P: u \rightsquigarrow v\left(\right.$ and $\operatorname{LD}_{B}(u, v)=\infty$ if no such $P$ exists),

2. $\operatorname{LD}_{B}(u, v) \leq \min _{P: u \rightsquigarrow v} \mathrm{wt}(P)$ where $P$ ranges over $\mathrm{U}$-shaped simple paths and simple cycles in $B$.

At the end of the computation, the returned value $c$ is the weight of a (generally nonsimple) cycle $C$, captured as a $\mathrm{U}$-shaped path on its smallest-level node. The cycle $C$ can be recovered by tracing backwards the updates of line 10 performed by the algorithm, starting from the node $x$ that performed the last update in line 13 . Hence, if $C$ traverses $k$ distinct edges, we can write

$$
c=\mathrm{wt}(C)=\sum_{i=1}^{k} k_{i} \cdot \mathrm{wt}\left(e_{i}\right)
$$

where each $e_{i}$ is a distinct edge, and $k_{i}$ is the number of times it appears in $C$.

Lemma 3. Let $h$ be the height of $\operatorname{Tree}(G)$. For every $k_{i}$ in Eq. (1), we have $k_{i} \leq 2^{h}$.

Proof. Note that the edge $e_{i}=\left(u_{i}, v_{i}\right)$ is first considered by MinCycle in the root bag $B_{i}$ of node $x_{i}$, where $x_{i}=\arg \max _{y_{i} \in\left\{u_{i}, v_{i}\right\}} \operatorname{Lv}\left(y_{i}\right)$ (line 10p. As MinCycle backtracks from $B_{i}$ to the root of Tree $(G)$, the edge $e_{i}$ can be traversed at most twice as many times

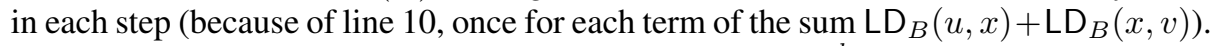
Hence, this doubling will occur at most $h$ times, and $k_{i} \leq 2^{h}$. 
Lemma 4. Let $c$ be the value returned by MinCycle, $h$ be the height of $\operatorname{Tree}(G)$, and $c^{*}=\min _{C} \operatorname{wt}(C)$ over all simple cycles $C$ in $G$. The following assertions hold:

1. If $G$ has no negative cycles, then $c=c^{*}$.

2. If $G$ has a negative cycle, then

(a) $c \leq c^{*}$.

(b) $|c|=O\left(\left|c^{*}\right| \cdot n \cdot 2^{h}\right)$.

Proof. By Remark 1 we have that $c^{*}=\operatorname{wt}(P)$ for a U-shaped path $P: x \rightsquigarrow x$. By Lemma 2, after MinCycle examines $B_{x}$, it will be $c \leq \operatorname{LD}_{B_{x}}(x, x) \leq c^{*}$, with the equalities holding if there are no negative cycles in $G$ (by the definition of $c^{*}$, as then $\mathrm{LD}_{B_{x}}(x, x)$ is witnessed by a simple cycle). By line $10, c$ can only decrease afterwards, and again by the definition of $c^{*}$ this can only happen if there are negative cycles in $G$. This proves items 1 and $2 \mathrm{a}$, and the remaining of the proof focuses on showing that $|c|=O\left(\left|c^{*}\right| \cdot n \cdot 2^{h}\right)$.

By rearranging the sum of Eq. (1), we can decompose the obtained cycle $C$ into a set of $k^{\prime+}$ non-negative cycles $C_{i}^{+}$, and a set of $k^{\prime-}$ negative cycles $C_{i}^{-}$, and each cycle $C_{i}^{+}$ and $C_{i}^{-}$appears with multiplicity $k_{i}^{+}$and $k_{i}^{-}$respectively. Then we have

$$
\begin{aligned}
|c| & =|\operatorname{wt}(C)|=\left|\sum_{i=1}^{k^{+}} k_{i}^{+} \cdot \operatorname{wt}\left(C_{i}^{+}\right)+\sum_{i=1}^{k^{\prime}} k_{i}^{-} \cdot \operatorname{wt}\left(C_{i}^{-}\right)\right| \leq\left|\sum_{i=1}^{k^{-}} k_{i}^{-} \cdot \operatorname{wt}\left(C_{i}^{-}\right)\right| \\
& \leq \sum_{i=1}^{k^{-}} k_{i}^{-} \cdot\left|\operatorname{wt}\left(C_{i}^{-}\right)\right| \leq\left|c^{*}\right| \cdot \sum_{i=1}^{k^{\prime-}} k_{i}^{-} \leq\left|c^{*}\right| \cdot \sum_{i=1}^{k} k_{i}^{-}=O\left(\left|c^{*}\right| \cdot n \cdot 2^{h}\right)
\end{aligned}
$$

The first inequality follows from $c<0$, the third inequality holds by the definition of $c^{*}$, and the last inequality holds since $k^{\prime-} \leq k$. Finally, we have $\sum_{i=1}^{k} k_{i}^{-}=O\left(n \cdot 2^{h}\right)$, since $k=O(n)$, and by Lemma 3 we have $k_{i}^{-} \leq 2^{h}$.

Next we discuss the time and space complexity of MinCycle.

Lemma 5. Let $h$ be the height of $\operatorname{Tree}(G)$. MinCycle accesses each bag of $\operatorname{Tree}(G) a$ constant number of times, and uses $O(h)$ additional space.

Proof. MinCycle accesses each bag a constant number of times, as it performs a postorder traversal on $\operatorname{Tree}(G)$ (line 2). Because it computes the local distances in a postorder manner, the number of local distance maps $\mathrm{LD}_{B}$ it remembers is bounded by the height $h$ of $\operatorname{Tree}(G)$. Since $\operatorname{Tree}(G)$ has constant width, $\operatorname{LD}_{B}$ requires a constant number of words for storing a constant number of nodes and weights in each $B$. Hence the total space usage is $O(h)$, and the result follows.

The following theorem summarizes the results of this section.

Theorem 2. Let $G=(V, E, \mathrm{wt})$ be a weighted graph of $n$ nodes with constant treewidth, and a balanced, binary tree-decomposition $\operatorname{Tree}(G)$ of $G$ be given. Let $c^{*}$, be the smallest weight of a simple cycle in G. Algorithm MinCycle uses $O(n)$ time and $O(\log n)$ additional space, and returns a value c such that: 
1. If $G$ has no negative cycles, then $c=c^{*}$.

2. If $G$ has a negative cycle, then

(a) $c \leq c^{*}$.

(b) $|c|=\left|c^{*}\right| \cdot n^{O(1)}$.

\section{The Minimum Ratio and Mean Cycle Problems}

In the current section we present algorithms for solving the minimum ratio and mean cycle problems for weighted graphs $G=\left(V, E, \mathrm{wt}, \mathrm{wt}^{\prime}\right)$ of constant treewidth.

Remark 2. If $G$ is not strongly connected we can compute its strongly connected components in linear time [44], and use the algorithms of this section to compute the minimum cycle mean $\nu_{i}^{*}$ in every component $\mathcal{G}_{i}$ separately. Afterwards, we compute $\nu^{*}(u)$ for every node $u$ by iteratively (i) finding the nodes $u$ that can reach the component $\mathcal{G}_{j}$ where $j=\operatorname{argmin}_{i} \nu_{i}^{*}$, (ii) assigning $\nu^{*}(u)=\nu_{j}^{*}$, and (iii) removing $\mathcal{G}_{j}$ and repeating. Since these operations require linear time, they do not impact the time complexity.

In light of Remark 2, we consider graphs that are strongly connected, and hence it follows that $\nu^{*}(u)$ is the same for every node $u$, and thus we will speak about the minimum ratio $\nu^{*}$ and mean $\mu^{*}$ values of $G$.

Claim 1. Let $\nu^{*}$ be the ratio value of $G$. Then $\nu^{*} \geq \nu$ iff for every cycle $C$ of $G$ we have $\mathrm{wt}_{\nu}(C) \geq 0$, where $\mathrm{wt}_{\nu}(e)=\mathrm{wt}(e)-\mathrm{wt}^{\prime}(e) \cdot \nu$ for each edge $e \in E$.

Proof. Indeed, for any cycle $C$ we have $\overline{\operatorname{wt}}(C) \geq \nu^{*} \geq \nu$. Then

$$
\begin{gathered}
\overline{\mathrm{wt}}(C) \geq \nu \Longleftrightarrow \overline{\mathrm{wt}}(C)-\nu \geq 0 \Longleftrightarrow \frac{\mathrm{wt}(C)-\nu \cdot \mathrm{wt}^{\prime}(C)}{\mathrm{wt}^{\prime}(C)} \geq 0 \\
\Longleftrightarrow \mathrm{wt}(C)-\nu \cdot \mathrm{wt}^{\prime}(C) \geq 0 \Longleftrightarrow \sum_{e \in C}\left(\mathrm{wt}(e)-\mathrm{wt}^{\prime}(e) \cdot \nu\right) \geq 0 \Longleftrightarrow \mathrm{wt}_{\nu}(C) \geq 0
\end{gathered}
$$

with the equality holding iff $\overline{\mathrm{wt}}(C)=\nu$.

Hence, given a tree-decomposition $\operatorname{Tree}(G)$, for any guess $\nu$ of the ratio value $\nu^{*}$, we can evaluate whether $\nu^{*} \geq \nu$ by constructing the weight function $\mathrm{wt}_{\nu}=\mathrm{wt}-\nu$ and executing algorithm MinCycle on input $G_{\nu}=\left(V, E, \mathrm{wt}_{\nu}\right)$. By Item 2a of Theorem 2 and Claim 1 we have that the returned value $c$ of MinCycle is $c \geq 0$ iff $\mathrm{wt}_{\nu}(C) \geq 0$ for all cycles $C$, iff $\nu^{*} \geq \nu$ (and in fact $c=0$ iff $\nu^{*}=\nu$ ).

Lemma 6. Let $G=\left(V, E, \mathrm{wt}, \mathrm{wt}^{\prime}\right)$ be a weighted graph of $n$ nodes with constant treewidth and minimum ratio value $\nu^{*}$. Let $\operatorname{Tree}(G)$ be a given balanced, binary treedecomposition of $G$ of constant width. For any rational $\nu$, the decision problem of whether $\nu^{*} \geq \nu\left(\right.$ or $\left.\nu^{*}=\nu\right)$ can be solved in $O(n)$ time and $O(\log n)$ extra space.

Proof. By Claim 11, we can test whether $\nu^{*} \geq \nu$ by testing whether $G_{\nu}=\left(V, E\right.$, wt $\left._{\nu}\right)$ has a negative cycle. By Theorem 2, a negative cycle in $G_{\nu}$ can be detected in $O(n)$ time and using $O(\log n)$ space. 


\subsection{Exact solution}

We now describe the method for determining the value $\nu^{*}$ of $G$ exactly. This is done by making various guesses $\nu$ such that $\nu^{*} \geq \nu$ and testing for negative cycles in the graph $G_{\nu}=\left(V, E, \mathrm{wt}_{\nu}\right)$. We first determine whether $\nu^{*}=0$, using Lemma 6 . In the remaining of this section we assume that $\nu^{*} \neq 0$.

Solution overview. Consider that $\nu^{*}>0$. First, we either find that $\nu^{*} \in(0,1)$ (hence $\left\lfloor\nu^{*}\right\rfloor=0$ ), or perform an exponential search of $O\left(\log \nu^{*}\right)$ iterations to determine $j \in$ $\mathbb{N}^{+}$such that $\nu^{*} \in\left[2^{j-1}, 2^{j}\right]$. In the latter case, we perform a binary search of $O\left(\log \nu^{*}\right)$ iterations in the interval $\left[2^{j-1}, 2^{j}\right]$ to determine $\left\lfloor\nu^{*}\right\rfloor$ (see Figure 2). Then, we can write $\nu^{*}=\left\lfloor\nu^{*}\right\rfloor+x$, where $x<1$ is an irreducible fraction $\frac{a^{\prime}}{b}$. It has been shown [41] that such $x$ can be determined by evaluating $O(\log b)$ inequalities of the form $x \geq \nu$. The case for $\nu^{*}<0$ is handled similarly.

Lemma 7. Let $\nu^{*} \neq 0$ be the ratio value of $G$. The value $\left\lfloor\nu^{*}\right\rfloor$ can be obtained by evaluating $O\left(\log \left|\nu^{*}\right|\right)$ inequalities of the form $\nu^{*} \geq \nu$.

Proof. First determine whether $\nu^{*}>0$, and assume w.l.o.g. that this is the case (the process is similar if $\left.\nu^{*}<0\right)$. Perform an exponential search on the interval $\left(0,2 \cdot\left\lfloor\nu^{*}\right\rfloor\right)$ by a sequence of evaluations of the inequality $\nu^{*} \geq \nu_{i}=2^{i}$. After $\log \left\lfloor\nu^{*}\right\rfloor+1$ steps we either have $\left\lfloor\nu^{*}\right\rfloor \in(0,1)$, or have determined a $j>0$ such that $\nu^{*} \in\left[\nu_{j-1}, \nu_{j}\right]$. Then, perform a binary search in the interval $\left[\nu_{j-1}, \nu_{j}\right]$, until the running interval $[\ell, r]$ has length at most 1 . Since $\nu_{j}-\nu_{j-1}=\nu_{j-1} \leq \nu^{*}$, this will happen after at most $\log \left\lceil\nu^{*}\right\rceil$ steps. Then either $\left\lfloor\nu^{*}\right\rfloor=\lfloor\ell\rfloor$ or $\left\lfloor\nu^{*}\right\rfloor=\lfloor r\rfloor$, which can be determined by evaluating the inequality $\nu^{*} \geq\lfloor r\rfloor$. A similar process can be carried out when $\nu^{*}<0$. Figure 2 shows an illustration of the search.

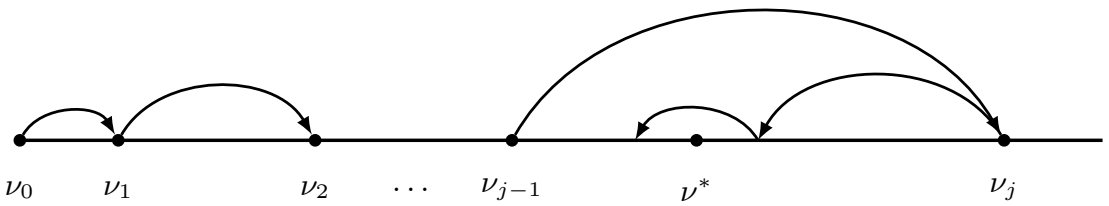

Fig. 2: Exponential search followed by a binary search to determine $\left\lfloor\nu^{*}\right\rfloor$

Let $T_{\max }=\max _{e} \mathrm{wt}^{\prime}(e)$ be the largest weight of an edge wrt $\mathrm{wt}^{\prime}$. Since $\nu^{*}$ is a number with denominator at most $(n-1) \cdot T_{\max }$, it can be determined exactly by carrying the binary search of Lemma 7 until the length of the running interval becomes at most $\frac{1}{\left((n-1) \cdot T_{\max }\right)^{2}}$ (thus containing a unique rational with denominator at most $(n-1)$. $\left.T_{\max }\right)$. Then $\nu^{*}$ can be obtained by using continued fractions, e.g. as in [36]. We rely in the work of Papadimitriou [41] to obtain a tighter bound.

Lemma 8. Let $\nu^{*} \neq 0$ be the ratio value of $G$, such that $\nu^{*}$ is the irreducible fraction $\frac{a}{b} \in(-1,1)$. Then $\nu^{*}$ can be determined by evaluating $O(\log b)$ inequalities of the form $\nu^{*} \geq \nu$. 
Proof. Consider that $\nu^{*}>0$ (the proof is similar when $\nu^{*}<0$ ). It is shown in [41] that a rational with denominator at most $b$ can be determined by evaluating $O(\log b)$ inequalities of the form $\nu^{*} \geq \nu$. We remark that $b$ is not required to be known, although the work of [41] assumes that a bound on the denominator of $\nu^{*}$ is known in advance.

Theorem 3. Let $G=\left(V, E, \mathrm{wt}, \mathrm{wt}^{\prime}\right)$ be a weighted graph of $n$ nodes with constant treewidth, and $\lambda=\max _{u}\left|a_{u} \cdot b_{u}\right|$ such that $\nu^{*}(u)$ is the irreducible fraction $\frac{a_{u}}{b_{u}}$. Let $\mathcal{T}(G)$ and $\mathcal{S}(G)$ denote the required time and space for constructing a balanced binary tree-decomposition $\operatorname{Tree}(G)$ of $G$ with constant width. The minimum ratio cycle problem for $G$ can be computed in

1. $O(\mathcal{T}(G)+n \cdot \log (\lambda))$ time and $O(\mathcal{S}(G)+n)$ space; and

2. $O(\mathcal{S}(G)+\log n)$ space.

Proof. In view of Remark 2 the graph $G$ is strongly connected and has a minimum ratio value $\nu^{*}$. Let $\nu^{*}=\left\lfloor\nu^{*}\right\rfloor+\frac{a^{\prime}}{b}$ with $\left|\frac{a^{\prime}}{b}\right|<1$. By Lemma $7,\left\lfloor\nu^{*}\right\rfloor$ can be determined by evaluating $O\left(\log \left|\nu^{*}\right|\right)=O(\log |a|)$ inequalities of the form $\nu^{*} \geq \nu$, and by Lemma 8 . $\frac{a^{\prime}}{b}$ can be determined by evaluating $O(b)$ such inequalities. A balanced binary treedecomposition Tree $(G)$ can be constructed once in $\mathcal{T}(G)$ time and $\mathcal{S}(G)$ space, and stored in $O(n)$ space. Tree $(G)$ is also a tree-decomposition of every $G_{\nu}$ required by Claim 1 By Theorem 2 a negative cycle in $G_{\nu}$ can be detected in $O(n)$ time and using $O(\log n)$ space. This concludes Item 1 . Item 2 is obtained by the same process, but with re-computing Tree $(G)$ every time MinCycle traverses from a bag to a neighbor (thus not storing Tree $(G)$ explicitly).

Using Theorem 1 we obtain from Theorem 3 the following corollary.

Corollary 1. Let $G=\left(V, E, \mathrm{wt}, \mathrm{wt}^{\prime}\right)$ be a weighted graph of $n$ nodes with constant treewidth, and $\lambda=\max _{u}\left|a_{u} \cdot b_{u}\right|$ such that $\nu^{*}(u)$ is the irreducible fraction $\frac{a_{u}}{b_{u}}$. The minimum ratio value problem for $G$ can be computed in

1. $O(n \cdot \log (\lambda \cdot n))$ time and $O(n)$ space; and

2. $O(\log n)$ space.

By setting $\mathrm{wt}^{\prime}(e)=1$ for each $e \in E$ in Corollary 1 we obtain the following corollary for the minimum mean cycle.

Corollary 2. Let $G=(V, E, w t)$ be a weighted graph of $n$ nodes with constant treewidth, and $\lambda=\max _{u}\left|\mu^{*}(u)\right|$. The minimum mean value problem for $G$ can be computed in

1. $O(n \cdot \log (\lambda \cdot n))$ time and $O(n)$ space; and

2. $O(\log n)$ space.

\subsection{Approximating the minimum mean cycle}

We now focus on the minimum mean cycle problem, and present algorithms for $\epsilon$ approximating the mean value $\mu^{*}$ of $G$ for any $0<\epsilon<1$ in $O(n \cdot \log (n / \epsilon))$ time, i.e., independent of $\mu^{*}$. 
Approximate solution in the absence of negative cycles. We first consider graphs $G$ that do not have negative cycles. Let $C$ be a minimum mean value cycle, and $C^{\prime}$ a minimum weight simple cycle in $G$, and note that $\mu^{*} \in\left[0, \mathrm{wt}\left(C^{\prime}\right)\right]$. Additionally, we have

$$
\mathrm{wt}\left(C^{\prime}\right) \leq \mathrm{wt}(C) \Longrightarrow \operatorname{wt}\left(C^{\prime}\right) \leq \frac{n}{|C|} \cdot \mathrm{wt}(C) \Longrightarrow \mathrm{wt}\left(C^{\prime}\right) \leq(n) \cdot \mu^{*}
$$

Consider a binary search in the interval $\left[0, \operatorname{wt}\left(C^{\prime}\right)\right]$, which in step $i$ approximates $\mu^{*}$ by the right endpoint $\mu_{i}$ of its current interval. The error is bounded by the length of the interval, hence $\mu_{i}-\mu^{*} \leq \mathrm{wt}\left(C^{\prime}\right) \cdot 2^{-i} \leq(n-1) \cdot \mu^{*} \cdot 2^{-i}$. To approximate within a factor $\epsilon$ we require

$$
2^{-i} \cdot(n-1) \leq \epsilon \Longrightarrow i \geq \log (n)+\log (1 / \epsilon)
$$

steps.

Remark 3. Note that for the minimum ratio value we have $\operatorname{wt}\left(C^{\prime}\right) \leq W^{\prime} \cdot n \cdot \nu^{*}$, where $W^{\prime}=\max _{e \in E} \mathrm{wt}^{\prime}(e)$. For $\epsilon$-approximating $\nu^{*}$ we would need $i \geq \log \left(n \cdot W^{\prime} / \epsilon\right)$ steps.

Approximate solution in the presence of negative cycles. We now turn our attention to $\epsilon$-approximating $\mu^{*}$ in the presence of negative cycles in $G$. Note that uniformly increasing the weight of each edge so that no negative edges exist does not suffice, as the error can be of order $\epsilon \cdot\left|W^{-}\right|$rather than $\epsilon \cdot \mu^{*}$, where $W^{-}$is the minimum edge weight.

Instead, let $c$ be the value returned by MinCycle on input $G$. Item $2 \mathrm{a}$ of Theorem 2 guarantees that for the weight function wt $-|c|(e)=\mathrm{wt}(e)+|c|$, the graph $G_{-|c|}=$ $\left(V, E, \mathrm{wt}_{-|c|}\right)$ has no negative cycles (although it might still have negative edges). The following lemma states that $\mu^{*}$ can be $\epsilon$-approximated by $\epsilon^{\prime}$-approximating the value $\mu^{\prime *}$ of $G_{-|c|}$, for some $\epsilon^{\prime}$ polynomially (in $n$ ) smaller than $\epsilon$.

Lemma 9. Let $\mu^{*}$ and $\mu^{\prime *}$ be the value of $G$ and $G_{-|c|}$ respectively, and $\epsilon$ some desired approximation factor of $\mu^{*}$, with $0<\epsilon<1$. There exists an $\epsilon^{\prime}=\epsilon / n^{O(1)}$ such that if $\mu^{\prime}$ is an $\epsilon^{\prime}$-approximation of $\mu^{\prime *}$ in $G_{-|c|}$, then $\mu=\mu^{\prime}-|c|$ is an $\epsilon$-approximation of $\mu^{*}$ in $G$.

Proof. By construction, we have $\mu^{\prime *}=\mu^{*}+|c|$, where $c$ defined above is the value returned by MinCycle on $G$. Let $c^{*}$ be the weight of a minimum-weight simple cycle in $G$. By Theorem 2 Item 2b, we have that $|c|=\left|c^{*}\right| \cdot n^{O(1)}$. Note that $\left|c^{*}\right| \leq(n-1) \cdot\left|\mu^{*}\right|$, hence $\mu^{\prime *}=\mu^{*}+\left|c^{*}\right| \cdot n^{O(1)} \leq\left|\mu^{*}\right| \cdot \alpha$ for $\alpha=n^{O(1)}$. Let $\epsilon^{\prime}=\epsilon / \alpha$. By $\epsilon^{\prime}$ approximating $\mu^{\prime *}$ by $\mu^{\prime}$ we have

$\left|\mu^{\prime}-\mu^{\prime *}\right| \leq \epsilon^{\prime} \cdot\left|\mu^{\prime *}\right| \Longrightarrow\left|\left(\mu^{\prime}-|c|\right)-\left(\mu^{\prime *}-|c|\right)\right| \leq \epsilon^{\prime} \cdot\left|\mu^{\prime *}\right| \Longrightarrow\left|\mu-\mu^{*}\right| \leq \epsilon^{\prime} \cdot\left|\mu^{*}\right| \cdot \alpha \leq \epsilon \cdot\left|\mu^{*}\right|$

The desired result follows.

Theorem 4. Let $G=(V, E, \mathrm{wt})$ be a weighted graph of $n$ nodes with constant treewidth. For any $0<\epsilon<1$, the minimum mean value problem can be $\epsilon$-approximated in $O(n \cdot \log (n / \epsilon))$ time and $O(n)$ space. 
Proof. In view of Remark 2 the graph $G$ is strongly connected and has a minimum mean value $\mu^{*}$. First, we construct a balanced binary tree-decomposition $\operatorname{Tree}(G)$ of $G$ in $O(n \cdot \log n)$ time and $O(n)$ space Theorem 1 . Let $c$ be the value returned by MinCycle on the input graph $G$. If $c \geq 0$, by Lemma 4 we have $\mu^{*} \geq 0$, and by Eq. (3) $\mu^{*}$ can be $\epsilon$-approximated in $O(\log (n / \epsilon))$ steps. If $c<0$, we construct the graph $G_{-|c|}=$ $\left(V, E, \mathrm{wt}_{-|c|}\right)$. By Lemma 9, $\mu^{*}$ can be $\epsilon$-approximated by $\epsilon^{\prime}$ approximating the mean value $\mu^{\prime *}$ of $G_{-|c|}$, where $\epsilon^{\prime}=\frac{\epsilon}{n^{O(1)}}$. By construction, $G_{-|c|}$ does not contain negative cycles, thus $\mu^{\prime *} \geq 0$, and by Eq. (3) $\mu^{\prime *}$ can be approximated in $O\left(\log \left(n / \epsilon^{\prime}\right)\right)=$ $O(\log (n / \epsilon))$ steps. By Lemma 5, each step requires $O(n)$ time. The statement follows.

\section{The Minimum Initial Credit Problem}

In the current section we present algorithms for solving the minimum initial credit problem on weighted graphs $G=(V, E, \mathrm{wt})$. We first deal with arbitrary graphs, and provide (i) an $O(n \cdot m)$ algorithm for the decision problem, and (ii) an $O\left(n^{2} \cdot m\right)$ for the value problem, improving the previously best upper-bounds. Afterwards we adapt our approach on graphs of constant treewidth to obtain an $O(n \cdot \log n)$ algorithm for the value problem.

Non-positive minimum initial credit. For technical convenience we focus on a variant of the minimum initial credit problem, where energies are non-positive, and the goal is to keep partial sums of path prefixes non-positive. Formally, given a weighted graph $G=(V, E, \mathrm{wt})$, the non-positive minimum initial credit value problem asks to determine for each node $u \in G$ the largest energy value $\mathrm{E}(u) \in \mathbb{Z}_{\leq 0} \cup\{-\infty\}$ with the following property: there exists an infinite path $\mathcal{P}=\left(u_{1}, u_{2} \ldots\right)$ with $u=u_{1}$, such that for every finite prefix $P$ of $\mathcal{P}$ we have $\mathrm{E}(u)+\mathrm{wt}(P) \leq 0$. Conventionally, we let $\mathrm{E}(u)=-\infty$ if no finite such value exists. The associated decision problem asks given a node $u$ and an initial credit $c \in \mathbb{Z}_{\leq 0}$ whether $\mathrm{E}(u) \geq c$. Hence, here minimality is wrt the absolute value of the energy. A solution to the standard minimum initial credit problem can be obtained by inverting the sign of each edge weight and solving the non-positive minimum initial credit problem in the resulting graph.

We start with some definitions and claims that will give the intuition for the algorithms to follow. First, we define the minimum initial credit of a pair of nodes $u, v$, which is the energy to reach $v$ from $u$ (i.e., the energy is wrt a finite path).

Finite minimum initial credit. For nodes $u, v \in V$, we denote with $\mathrm{E}_{v}(u) \in \mathbb{Z}_{\leq 0} \cup$ $\{-\infty\}$ the largest value with the following property: there exists a path $P: u \rightsquigarrow v$ such that for every prefix $P^{\prime}$ of $P$ we have $\mathrm{E}_{v}(u)+\operatorname{wt}\left(P^{\prime}\right) \leq 0$. Note that for every pair of nodes $u, v \in V$, we have $\mathrm{E}(u) \geq \mathrm{E}_{v}(u)+\mathrm{E}(v)$. Conventionally, we let $\mathrm{E}_{v}(u)=-\infty$ if no such value exists (i.e., there is no path $u \rightsquigarrow v$ ).

Remark 4. For any $u \in V$, let $P: u \rightsquigarrow v$ be a witness path for $\mathrm{E}_{v}(u)>-\infty$. Then

$$
\mathrm{E}_{v}(u)+\operatorname{wt}(P) \leq 0 \Longrightarrow \mathrm{E}_{v}(u) \leq-\operatorname{wt}(P) \leq-d(u, v)
$$

i.e., the energy to reach $v$ from $u$ is upper bounded by minus the distance from $u$ to $v$.

Highest-energy nodes. Given a (possibly infinite) path $P$ with $\operatorname{wt}(P)<\infty$, we say that a node $x \in P$ is a highest-energy node of $P$ if there exists a highest-energy prefix 
$P_{1}$ of $P$ ending in $x$ such that for any prefix $P_{2}$ of $P$ we have wt $\left(P_{1}\right) \geq \operatorname{wt}\left(P_{2}\right)$. Note that since the weights are integers, for every pair of paths $P_{1}^{\prime}, P_{2}^{\prime}$, it is either $\left|w t\left(P_{1}^{\prime}\right)-\operatorname{wt}\left(P_{2}^{\prime}\right)\right|=0$ or $\left|w t\left(P_{1}^{\prime}\right)-w t\left(P_{2}^{\prime}\right)\right| \geq 1$. Therefore the set $\left\{w t\left(P_{i}\right)\right\}_{i}$ of weights of prefixes of $P$ has a maximum, and thus a highest-energy node always exists when $\operatorname{wt}(P)<\infty$. The following properties are easy to verify:

1. If $x$ is a highest-energy node in a path $P: u \rightsquigarrow v$, then $\mathrm{E}_{v}(x)=0$.

2. If $x$ is a highest-energy node in an infinite path $\mathcal{P}$, then $\mathrm{E}(x)=0$.

The following claim states that the energy $\mathrm{E}(u)$ of a node $u$ is the maximum energy $\mathrm{E}_{v}(u)$ to reach a 0 -energy node $v$.

Claim 2. For every $u \in V$, we have $\mathrm{E}(u)=\max _{v: \mathrm{E}(v)=0} \mathrm{E}_{v}(u)$.

Proof. The direction $\mathrm{E}(u) \geq \max _{v: \mathrm{E}(v)=0} \mathrm{E}_{v}(u)$ is straightforward. For the other direction, consider that $\mathrm{E}(u)>-\infty$ (trivially, $-\infty \leq \max _{v: \mathrm{E}(v)=0} \mathrm{E}_{v}(u)$ ) and let $\mathcal{P}$ be a witness path for $\mathrm{E}(u)$. Since $\mathrm{E}(u)>-\infty$, we have wt $(\mathcal{P})<\infty$, and $\mathcal{P}$ has some highest-energy node $x$, thus $\mathrm{E}(x)=0$. Since $x$ is on the witness $\mathcal{P}$ of $\mathrm{E}(u)$, we have $\mathrm{E}(u) \leq \mathrm{E}_{x}(u) \leq \max _{v: \mathrm{E}(v)=0} \mathrm{E}_{v}(u)$. The result follows.

\subsection{The decision problem for general graphs}

Here we address the decision problem, namely, given some node $u \in V$ and an initial credit $c \in \mathbb{Z}_{\leq 0}$, determine whether $\mathrm{E}(u) \geq c$. The following claim states that if $\mathrm{E}(u) \geq$ $c$, then a non-positive cycle can be reached from $u$ with initial credit $c$, by paths of length less than $n$.

Claim 3. For every $u \in V$ and $c \in \mathbb{Z}_{\leq 0}$, we have that $\mathrm{E}(u) \geq c$ iff there exists a simple cycle $C$ such that (i) wt $(C) \leq 0$ and (ii) for every $v \in C$ we have that $\mathrm{E}_{v}(u) \geq c$, which is witnessed by a path $P_{v}: u \rightsquigarrow v$ with $\left|P_{v}\right|<n$.

Proof. For the one direction, if wt $(C) \leq 0$ we have wt $\left(C^{\omega}\right)<\infty$, thus $C$ contains a 0-energy node $w$. By Claim 2. $\mathrm{E}(u)=\max _{v: \mathrm{E}(v)=0} \mathrm{E}_{v}(u) \geq \mathrm{E}_{w}(u) \geq c$. For the other direction, let $\mathcal{P}$ be a witness path for $\mathrm{E}(u)$, and we can assume w.l.o.g. that $\mathcal{P}$ does not contain positive cycles. Then for every prefix $P_{v}: u \rightsquigarrow v$ of $\mathcal{P}$ we have $\mathrm{E}(u)+\operatorname{wt}\left(P_{v}\right) \leq 0$, thus $\mathrm{E}_{v}(u) \geq \mathrm{E}(u) \geq c$, and the $n$-th such prefix contains a nonpositive cycle $C$. The result follows.

Algorithm DecisionEnergy. Claim 3 suggests a way to decide whether $\mathrm{E}(u) \geq c$. First, we start with energy $c$ from $u$, and perform a sequence of $n-1$ relaxation steps, similar to the Bellman-Ford algorithm, to discover the set $V_{u}^{c}$ of nodes that can be reached from $u$ with initial credit $c$ by a path of length at most $n-1$. Afterwards, we perform a Bellman-Ford computation on the subgraph $G \nmid V_{u}^{c}$ induced by the set $V_{u}^{c}$. By Claim 3, we have that $\mathrm{E}(u) \geq c$ iff $G \uparrow V_{u}^{c}$ contains a non-positive cycle. Algorithm 2 (DecisionEnergy) gives a formal description. The for loop in lines 6 12 is similar to the procedure ROUND from the algorithm of [9].

Detecting non-positive cycles. It is known that the Bellman-Ford algorithm can detect negative cycles. To detect non-positive cycles in a graph $G$ with $n$ nodes and weight function wt, we execute Bellman-Ford on $G$ with a slightly modified weight function 
$\mathrm{wt}^{\prime}$ for which $\mathrm{wt}^{\prime}(e)=\mathrm{wt}(e)-\frac{1}{n}$. Then for any simple cycle $C$ in $G$ we have $\operatorname{wt}(C) \leq$ 0 iff $\mathrm{wt}^{\prime}(C)<0$. Indeed,

$$
\mathrm{wt}^{\prime}(C)<0 \Longleftrightarrow \sum_{e \in C} \mathrm{wt}(e)-\sum_{e \in C} \frac{1}{n}<0 \Longleftrightarrow \mathrm{wt}(C)<\frac{|C|}{n} \Longleftrightarrow \mathrm{wt}(C) \leq 0
$$

since $|C| \leq n$ and $\operatorname{wt}(C) \in \mathbb{Z}$.

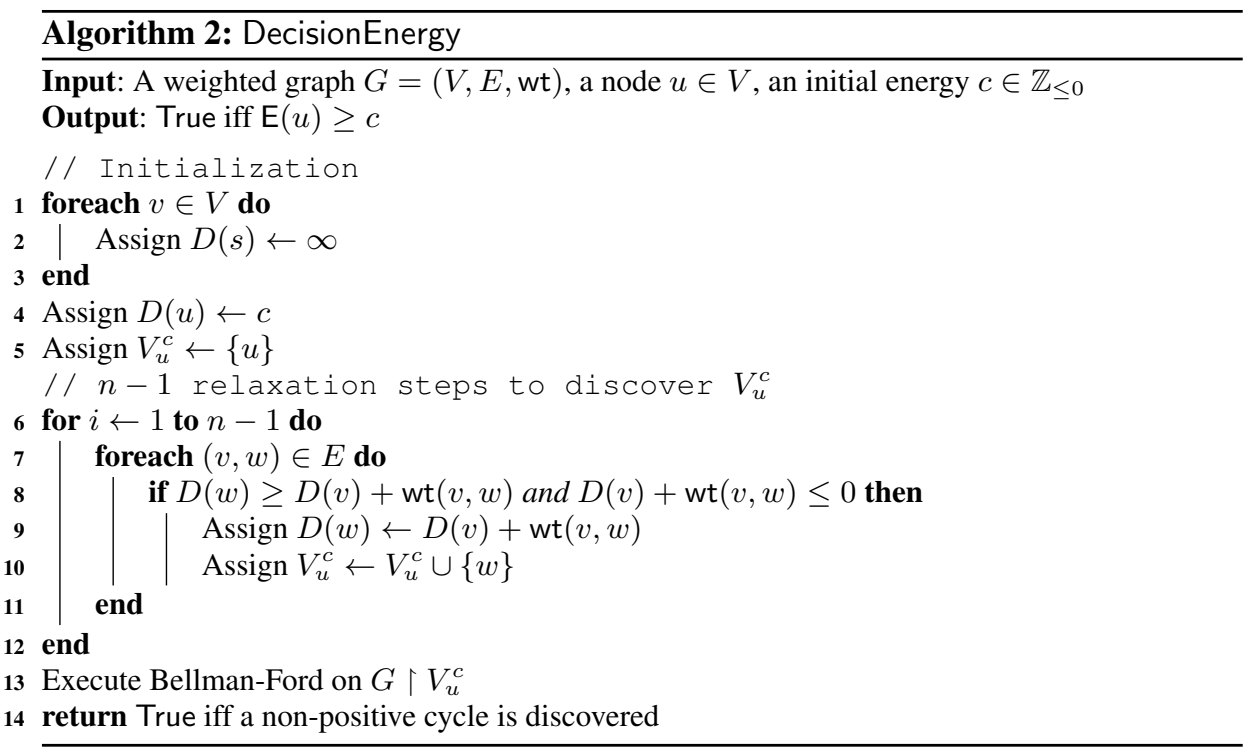

The correctness of DecisionEnergy follows directly from Claim 3 . The time complexity is $O(n \cdot m)$ time spent in the for loop of lines 6 12, plus $O(n \cdot m)$ time for the BellmanFord. We thus obtain the following theorem.

Theorem 5. Let $G=(V, E$, wt $)$ be a weighted graph of $n$ nodes and $m$ edges. Let $u \in V$ be an initial node, and $c \in \mathbb{Z}_{\leq 0}$ be an initial credit. The decision problem of whether $\mathrm{E}(u) \geq c$ can be solved in $O(n \cdot m)$ time and $O(n)$ space.

\subsection{The value problem for general graphs}

We now turn our attention to the value version of the minimum initial credit problem, where the task is to determine $\mathrm{E}(u)$ for every node $u$. The following claim establishes that if for all energies to reach some node $v$ we have $\mathrm{E}_{v}(w)<0$, then $\mathrm{E}_{v}(u)=-d(u, v)$, i.e., the energy to reach $v$ from every node $u$ is minus the distance from $u$ to $v$.

Claim 4. If for all $w \in V \backslash\{v\}$ we have $\mathrm{E}_{v}(w)<0$, then for each $u \in V \backslash\{v\}$ we have $\mathrm{E}_{v}(u)=-d(u, v)$. 
Proof. Let $P: u \rightsquigarrow v$ be a witness path to the distance, i.e., $\operatorname{wt}(P)=d(u, v)<\infty$ (if $d(u, v)=\infty$ the statement is trivially true). Since every highest-energy node $x$ of $P$ has $\mathrm{E}_{v}(x)=0$, we have that $x=v$. Hence, $P$ is a highest-energy prefix of itself, and for each prefix $P^{\prime}$ of $P$ we have $-\operatorname{wt}(P)+\operatorname{wt}\left(P^{\prime}\right) \leq 0$ and thus $\mathrm{E}_{v}(u) \geq-\operatorname{wt}(P)=$ $-d(u, v)$. By Remark 4 it is $\mathrm{E}_{v}(u) \leq-d(u, v)$. The result follows.

An $O\left(n^{2} \cdot m\right)$ time solution to the value problem. Claim 4 together with Theorem 5 lead to an $O\left(n^{2} \cdot m\right)$ method for solving the minimum initial credit value problem. First, we compute the set $X=\{v \in V: \mathrm{E}(v)=0\}$ in $O\left(n^{2} \cdot m\right)$ time, by testing whether $\mathrm{E}(u) \geq 0$ for each node $u$. Afterwards, we contract the set $X$ to a new node $z$, and by Claim 2 for every remaining node $u$ we have $\mathrm{E}(u)=\max _{v \in X} \mathrm{E}_{v}(u)=\mathrm{E}_{z}(u)$. Since $u \notin X$, the energy of $u$ is strictly negative, and thus $\mathrm{E}_{z}(u)<0$. Finally, by Claim 4 , we have $\mathrm{E}_{z}(u)=-d(u, z)$. Hence it suffices to compute the distance of each node $u$ to $z$, which can be obtained in $O(n \cdot m)$ time.

In the remaining of this subsection we provide a refined solution of $O(k \cdot n \cdot m)$ time, where $k=|X|+1$ is the number of 0-energy nodes (plus one). Hence this solution is faster in graphs where $k=o(n)$. This is achieved by algorithm ZeroEnergyNodes for computing the set $X$ faster.

Determining the 0 -energy nodes. The first step for solving the minimum initial credit problem is determining the set $X$ of all 0-energy nodes of $G$. To achieve this, we construct the graph $G_{2}=\left(V_{2}, E_{2}\right.$, wt $\left.t_{2}\right)$ with a fresh node $z \notin V$ as follows:

1. The node set is $V_{2}=V \cup\{z\}$,

2. The edge set is $E_{2}=E \cup(\{z\} \times V)$,

3. The weight function wt $t_{2}: E_{2} \rightarrow \mathbb{Z}$ is

$$
\operatorname{wt}_{2}(u, v)= \begin{cases}0 & \text { if } u=z \\ \operatorname{wt}(u, v) & \text { otherwise }\end{cases}
$$

Remark 5. Since for every outgoing edge $(z, x)$ of $z$ we have $\mathrm{wt}_{2}(z, x)=0$, if $z$ is a highest-energy node in a path of $G_{2}$, so is $x$. Hence every non-positive cycle in $G_{2}$ has a highest-energy node other than $z$.

Note that for every $u \in V$, the energy $\mathrm{E}(u)$ is the same in $G$ and $G_{2}$.

Algorithm ZeroEnergyNodes. Algorithm 3 describes ZeroEnergyNodes for obtaining the set of all 0-energy nodes in $G_{2}$. Informally, the algorithm performs a sequence of modifications on a graph $\mathcal{G}$, initially identical to $G_{2}$. In each step, the algorithm executes a Bellman-Ford computation on the current graph $\mathcal{G}$ with $z$ as the source node, as long as a non-positive cycle $C$ is discovered. For every such $C$, it determines a highestenergy node $w$ of $C$, and modifies $\mathcal{G}$ by replacing every incoming edge $(x, w)$ with an edge $(x, z)$ of the same weight, and then removing $w$. See Figure 3 for an illustration.

As 0-energy nodes are discovered, ZeroEnergyNodes performs a sequence of modifications to the graph $\mathcal{G}$. We denote with $\mathcal{G}^{k}$ the graph $\mathcal{G}$ after the $k$-th node has been added to $X$ (and $\mathcal{G}^{0}=G_{2}$ ). We also use the superscript- $k$ in our graph notation to make it specific to $\mathcal{G}^{k}$ (e.g. $d^{k}(u, z)$ and $\mathrm{E}_{z}^{k}(u)$ denote respectively the distance from $u$ to $z$, and the energy to reach $z$ from $u$ in $\mathcal{G}^{k}$ ). The following two lemmas establish the correctness of ZeroEnergyNodes.

Lemma 10. For every $w \in X$ we have $\mathrm{E}(w)=0$. 


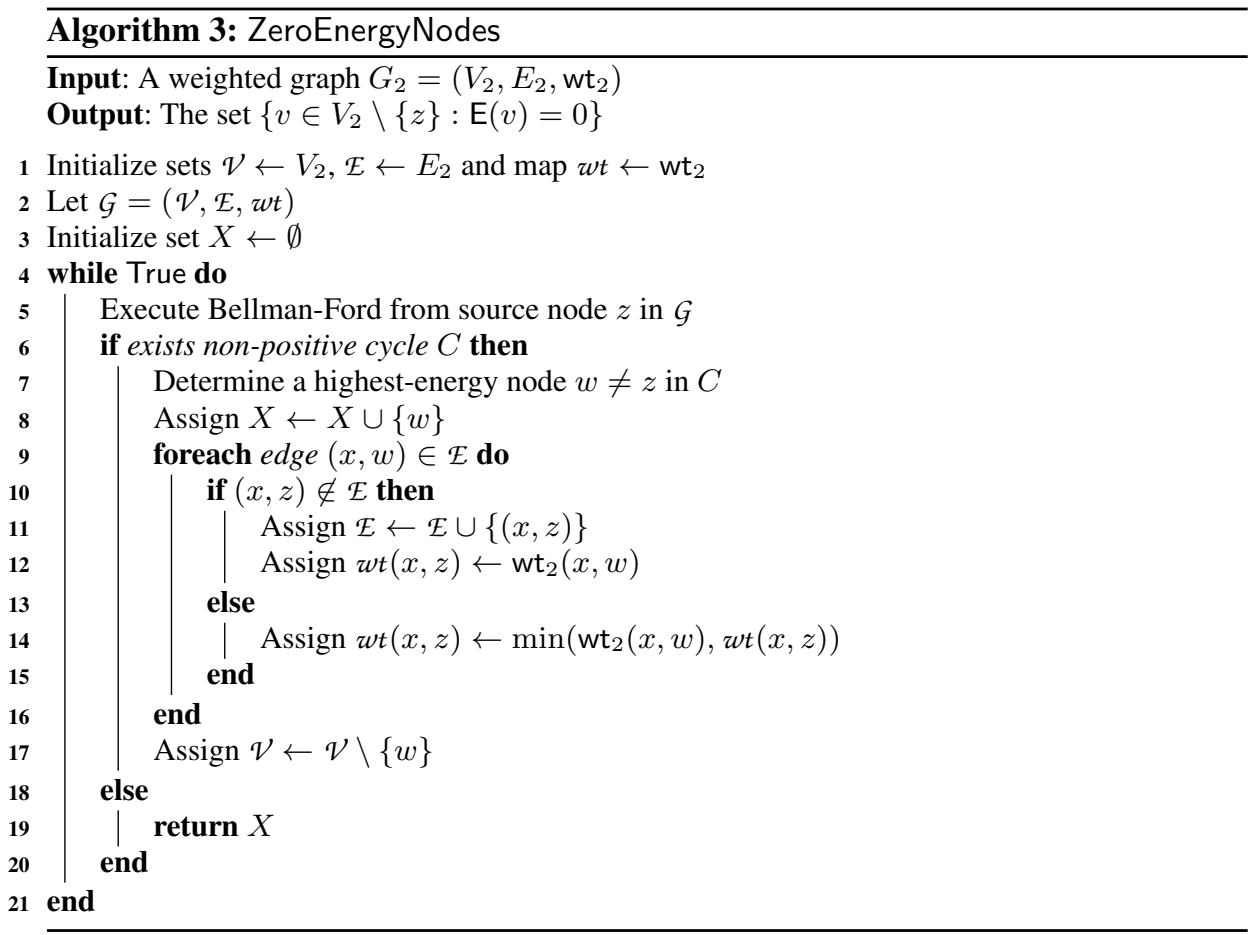

Proof. The proof is by induction on the size of $X$. It is trivially true when $|X|=0$. For the inductive step, let $w$ be the $k+1$-th node added in $X$. By line $7, w$ is a highest-energy node in a non-positive cycle $C$ of $\mathcal{G}^{k}$. We split into two cases.

1. If $z \notin C$, then $C$ is also a cycle of $G$, hence $w$ is a highest-energy node in the infinite path $\mathcal{P}=C^{\omega}$ of $G$, and $\mathrm{E}(w)=0$.

2. If $z \in C$, let $x$ be the node before $z$ in $C$. By the modifications of lines 11 and 14 , it is $w t^{k}(x, z)=\mathrm{wt}_{2}\left(x, w^{\prime}\right)$, where $w^{\prime}$ is a node that has been added to $X$ when the algorithm run on $\mathcal{G}^{i}$ for some $i<k$. It follows that $w$ is a highest-energy node in a path $P: z \rightsquigarrow w^{\prime}$ in $G_{2}$, and thus a highest-energy node in a suffix $P^{\prime}: w \rightsquigarrow w^{\prime}$ of $P$, where $P^{\prime}$ is a path in $G$. Hence $\mathrm{E}_{w^{\prime}}(w)=0$. By the induction hypothesis, $w^{\prime}$ is a 0 -energy node, i.e., $\mathrm{E}\left(w^{\prime}\right)=0$, thus by Claim 2 we have $\mathrm{E}(w) \geq \mathrm{E}_{w^{\prime}}(w)=0$.

The result follows.

Lemma 11. For every $w \in V: \mathrm{E}(w)=0$ we have $w \in X$.

Proof. Consider any $w \in V: \mathrm{E}(w)=0$. For some $i \in \mathbb{N}$, we say that $\mathcal{G}^{i}$ "is aware of $w$ " if either $\mathcal{G}^{i}$ has a non-positive cycle $C: w \rightsquigarrow w$, or $w \in X$ when $|X|=i$. Note that when ZeroEnergyNodes terminates there are no non-positive cycles in $\mathcal{G}^{|X|}$. Hence, it suffices to argue that there exists a $k \in \mathbb{N}$ such that for each $i \geq k, \mathcal{G}^{i}$ is aware of $w$. We first argue that there exists a $k$ for which $\mathcal{G}^{k}$ is aware of $w$.

Let $\mathcal{P}$ be a witness for $\mathrm{E}(w)=0$, hence $\mathcal{P}$ traverses a non-positive cycle $C_{1}$ in $G$, thus $C_{1}$ exists in $\mathcal{G}^{0}$. Then there exists a smallest $j \in \mathbb{N}$ such that some node $w^{\prime}$ of $\mathcal{P}$ is identified as a highest-energy node in a non-positive cycle $C_{2}$ (possibly $C_{1}=C_{2}$ ), and 
inserted to $X$. If $w=w^{\prime}$, we have that $\mathcal{G}^{j}$ is aware of $w$. Otherwise, since $\mathrm{E}(w)=0$ and $w^{\prime}$ is a node in the witness $\mathcal{P}$, we have $\mathrm{E}_{w^{\prime}}(w)=0$. By the choice of $w^{\prime}$, the path $\mathcal{P}$ exists in $\mathcal{G}^{j}$, therefore $\mathrm{E}_{w^{\prime}}^{j}(w)=\mathrm{E}_{w^{\prime}}(w)=0$, and by Remark 4 , we have $d^{j}\left(w, w^{\prime}\right) \leq 0$. It is straightforward that after the modifications in lines 11 and 14 , we have that $d^{j+1}(w, z) \leq d^{j}\left(w, w^{\prime}\right) \leq 0$, and since $w^{j}(z, w)=\mathrm{wt}_{2}(z, w)=0$, we have a non-positive cycle $C: w \rightsquigarrow w$ in $\mathcal{G}^{j+1}$ through $z$. Hence either $\mathcal{G}^{j}$ or $\mathcal{G}^{j+1}$ is aware of $w$, thus there exists a $k \in \mathbb{N}$ for which $\mathcal{G}^{k}$ is aware of $w$.

Finally, observe that the distance $d^{i}(w, z)$ does not increase in any $\mathcal{G}^{i}$ for $i \geq k$ until $w$ is inserted to $X$, hence for each $i \geq k$, the graph $\mathcal{G}^{i}$ is aware of $w$. The desired result follows.

Determining the negative-energy nodes. Having computed the set $X$ of all the 0 energy nodes of $G$, the second step for solving the minimum initial value credit problem is to determine the energy of every other node $u \in V \backslash X$. Recall the graph $\mathcal{G}^{|X|}=$ $\left(\mathcal{V}^{|X|}, \mathcal{E}^{|X|}, w t^{|X|}\right)$ after the end of ZeroEnergyNodes.

Lemma 12. For every $u \in V \backslash X$ we have $\mathrm{E}(u)=-d^{|X|}(u, z)$.

Proof. Consider any node $u \in V \backslash X=\mathcal{V}^{|X|} \backslash\{z\}$. By Claim 4, in the graph $G$ we have $\mathrm{E}(u)=\max _{v: \mathrm{E}(v)=0} \mathrm{E}_{v}(u)$, and by the correctness of ZeroEnergyNodes from Lemma 10 and Lemma 11 we have $X=\{v: \mathrm{E}(v)=0\}$, thus $\mathrm{E}(u)=$ $\max _{v \in X} \mathrm{E}_{v}(u)$. It is straightforward to verify that at the end of ZeroEnergyNodes, we have $\max _{v \in X} \mathrm{E}_{v}(u)=\mathrm{E}_{z}^{|X|}(u)$, i.e., the maximum energy to reach the set $X$ in $G$ is the energy to reach $z$ in $\mathcal{G}^{|X|}$. For all $v \in \mathcal{V}^{|X|} \backslash\{z\}$ it is $\mathrm{E}_{z}^{|X|}(v)<0$, otherwise we would have $\mathrm{E}(v)=0$ and thus $v \in X$ and $v \notin \mathcal{V}^{|X|}$. Then by Claim 4 . $\mathrm{E}_{z}^{|X|}(u)=-d^{|X|}(u, z)$. We conclude that $\mathrm{E}(u)=-d^{|X|}(u, z)$.

Hence, to compute the energy $\mathrm{E}(u)$ of every node $u \in V \backslash X$, it suffices to compute its distance to $z$ in $\mathcal{G}^{|X|}$. This is straightforward by reversing the edges of $\mathcal{G}^{|X|}$ and performing a Bellman-Ford computation with $z$ as the source node. Figure 3 illustrates the algorithms on a small example. We obtain the following theorem.

Theorem 6. Let $G=(V, E, \mathrm{wt})$ be a weighted graph of $n$ nodes and $m$ edges, and $k=|\{v \in V: \mathrm{E}(v)=0\}|+1$. The minimum initial credit value problem for $G$ can be solved in $O(k \cdot n \cdot m)$ time and $O(n)$ space.

Proof. Lemma 10, Lemma 11 and Lemma 12 establish the correctness, so it remains to argue about the complexity. The while block of line 4 is executed at most once for each 0 -energy node, hence at most $k$ times. Inside the block, the execution of Bellman-Ford in line 5 requires $O(n \cdot m)$ time and $O(m)$ space. Since the Bellman-Ford algorithm uses backpointers to remember predecessors of nodes in distances, a highest-energy node $w$ of a non-positive cycle $C$ in line $7 \mathrm{can}$ be determined in $O(n)$. Finally, the for loop of line 9 will consider each edge $(x, w)$ at most once, hence it requires $O(m)$ for all iterations of the while loop. Thus ZeroEnergyNodes uses $O(k \cdot n \cdot m)$ time and $O(n)$ space in total. The last execution of Bellman-Ford to determine the energy of negativeenergy nodes does not affect the complexity. The result follows.

Corollary 3. Let $G=(V, E, \mathrm{wt})$ be a weighted graph of $n$ nodes and $m$ edges. The minimum initial credit value problem for $G$ can be solved in $O\left(n^{2} \cdot m\right)$ time and $O(n)$ space. 


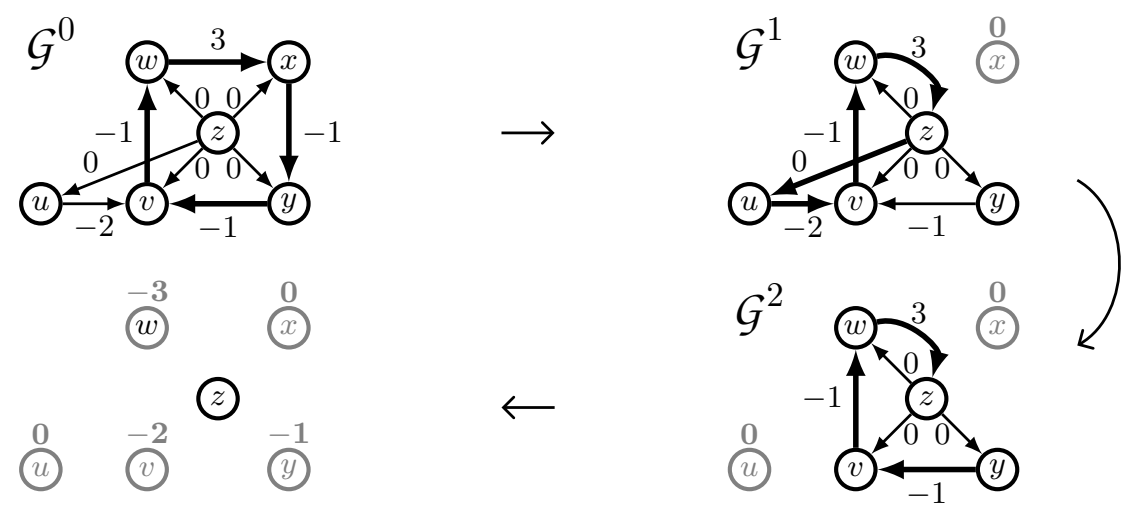

Fig. 3: Solving the value problem using operations on the graph $\mathcal{G}$. Initially we examine $\mathcal{G}^{0}$, and a non-positive cycle is found (boldface edges) with highest-energy node $x$. Thus $\mathrm{E}(x)=0$, and we proceed with $\mathcal{G}^{1}$, to discover $\mathrm{E}(u)=0$. In $\mathcal{G}^{2}$ all cycles are positive, and the energy of each remaining node is minus its distance to $z$.

\subsection{The value problem for constant-treewidth graphs}

We now turn our attention to the minimum initial credit value problem for constanttreewidth graphs $G=(V, E, \mathrm{wt})$. Note that in such graphs $m=O(n)$, thus Theorem 6 gives an $O\left(n^{3}\right)$ time solution as compared to the existing $O\left(n^{4} \cdot \log (n \cdot W)\right)$ time solution. This section shows that we can do significantly better, namely reduce the time complexity to $O(n \cdot \log n)$. This is mainly achieved by algorithm ZeroEnergyNodesTW for computing the set $X$ of 0 -energy nodes fast in constant-treewidth graphs.

Extended + and min operators. Recall the graph $G_{2}=\left(V_{2}, E_{2}, \mathrm{wt}_{2}\right)$ from the last section. Given Tree $(G)$, a balanced and binary tree-decomposition Tree $\left(G_{2}\right)$ of $G_{2}$ with width increased by 1 can be easily constructed by (i) inserting $z$ to every bag of Tree $(G)$, and (ii) adding a new root bag that contains only $z$. Let $\mathcal{I}=\mathbb{Z} \times V \times \mathbb{Z}$. For a map $f: V_{2} \times V_{2} \rightarrow \mathbb{Z}$, define the map $g_{f}: V_{2} \times V_{2} \rightarrow \mathcal{I}$ as

$$
g_{f}(u, v)= \begin{cases}(f(u, v), u, 0) & \text { if } f(u, v)<0 \text { or } v=z \\ (f(u, v), v, f(u, v)) & \text { otherwise }\end{cases}
$$

and for triplets of elements $\alpha_{1}=\left(a_{1}, b_{1}, c_{1}\right), \alpha_{2}=\left(a_{2}, b_{2}, c_{2}\right) \in \mathcal{I}$, define the operations

1. $\min \left(\alpha_{1}, \alpha_{2}\right)=\alpha_{i}$ with $i=\arg \min _{j \in\{1,2\}} a_{j}$

2. $\alpha_{1}+\alpha_{2}=\left(a_{1}+a_{2}, b, c\right)$, where $c=\max \left(c_{1}, a_{1}+c_{2}\right)$ and $b=b_{1}$ if $c=c_{1}$ else $b=b_{2}$.

In words, if $f$ is a weight function, then $g_{f}(u, v)$ selects the weight of the edge $(u, v)$, and its highest-energy node (i.e., $u$ if $f(u, v)<0$, and $v$ otherwise, except when $v=z$ ), together with the weight to reach that highest energy node node from $u$. Recall that algorithm MinCycle from Section 3 traverses a tree-decomposition bottom-up, and for each encountered bag $B$ stores a map $\operatorname{LD}_{B}$ such that $\operatorname{LD}_{B}(u, v)$ is upper bounded by the weight of the shortest $\mathrm{U}$-shaped simple path $u \rightsquigarrow v$ (or simple cycle, if $u=v$ ). Our algorithm ZeroEnergyNodesTW for determining all 0-energy nodes is similar, only that now $\mathrm{LD}_{B}$ stores triplets $(a, b, c)$ where $a$ is the weight of a $\mathrm{U}$-shaped path $P, b$ is 
a highest-energy node of $P$, and $c$ the weight of a highest-energy prefix of $P$. For two triplets $\alpha_{1}=\left(a_{1}, b_{1}, c_{1}\right), \alpha_{2}=\left(a_{2}, b_{2}, c_{2}\right) \in \mathcal{I}$ corresponding to $\mathrm{U}$-shaped paths $P_{1}$ and $P_{2}, \min \left(\alpha_{1}, \alpha_{2}\right)$ selects the path with the smallest weight, and $\alpha_{1}+\alpha_{2}$ determines the weight, a highest-energy node, and the weight of a highest-energy prefix of the path $P_{1} \circ P_{2}$ (see Figure 4 ).

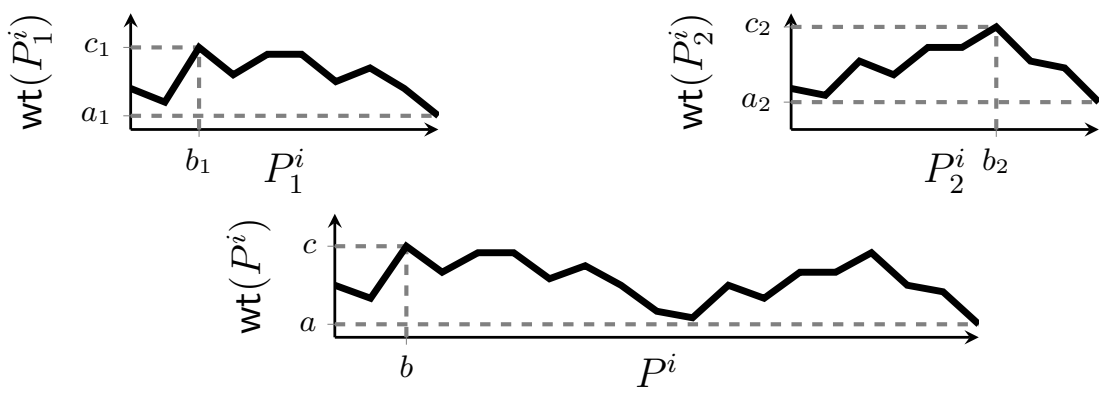

Fig. 4: Illustration of the $\alpha_{1}+\alpha_{2}$ operation, corresponding to concatenating paths $P_{1}$ and $P_{2}$. The path $P_{j}^{i}$ denotes the $i$-th prefix of $P_{j}$. We have $P=P_{1} \circ P_{2}$, and the corresponding tripplet $\alpha=(a, b, c)$ denotes the weight $a$ of $P$, its highest-energy node $b$, and the weight $c$ of a highest-energy prefix.

Algorithm ZeroEnergyNodesTW. The algorithm ZeroEnergyNodesTW for computing the set of 0-energy nodes in constant-treewidth graphs follows the same principle as ZeroEnergyNodes for general graphs. It stores a map of edge weights $w t: E_{2} \rightarrow \mathbb{Z} \cup$ $\{\infty\}$, and initially $w t(u, v)=\mathrm{wt}_{2}(u, v)$ for each $(u, v) \in E_{2}$. The algorithm performs a bottom-up pass, and computes in each bag the local distance map $\operatorname{LD}_{B}: B \times B \rightarrow \mathcal{I}$ that captures $\mathrm{U}$-shaped $u \rightsquigarrow v$ paths, together with their highest-energy nodes. When a non-positive cycle $C$ is found in some bag $B$, the method KillCycle is called to modify the edges of a highest-energy node $w$ of $C$ and its incoming neighbors by updating the map wt. These updates generally affect the distances between the rest of the nodes in the graph, hence some local distance maps $\operatorname{LD}_{B}$ need to be corrected. However, each such edge modification only affects the local distance map of bags that appear in a path from a bag $B^{\prime}$ to some ancestor $B^{\prime \prime}$ of $B^{\prime}$. Instead of restarting the computation as in ZeroEnergyNodes, the method Update is called to correct those local distance maps along the path $B^{\prime} \rightsquigarrow B^{\prime \prime}$.

The following lemma establishes the correctness of ZeroEnergyNodesTW. Similarly as for Lemma 10 and Lemma 11 we denote with $\mathcal{G}^{k}$ the graph obtained by considering the edges $(u, v)$ for which $w t(u, v)<\infty$ when $|X|=k$.

Lemma 13. For every $v \in V \backslash\{z\}$ we have $v \in X$ iff $\mathrm{E}(v)=0$.

Proof. We only need to argue that ZeroEnergyNodesTW correctly computes the nonpositive cycles in every $\mathcal{G}^{k}$, as then the correctness follows from the correctness Lemma 10 and Lemma 11 of ZeroEnergyNodes. Since by Remark 1 every cycle is a U-shaped path in some bag, it suffices to argue that whenever ZeroEnergyNodesTW 

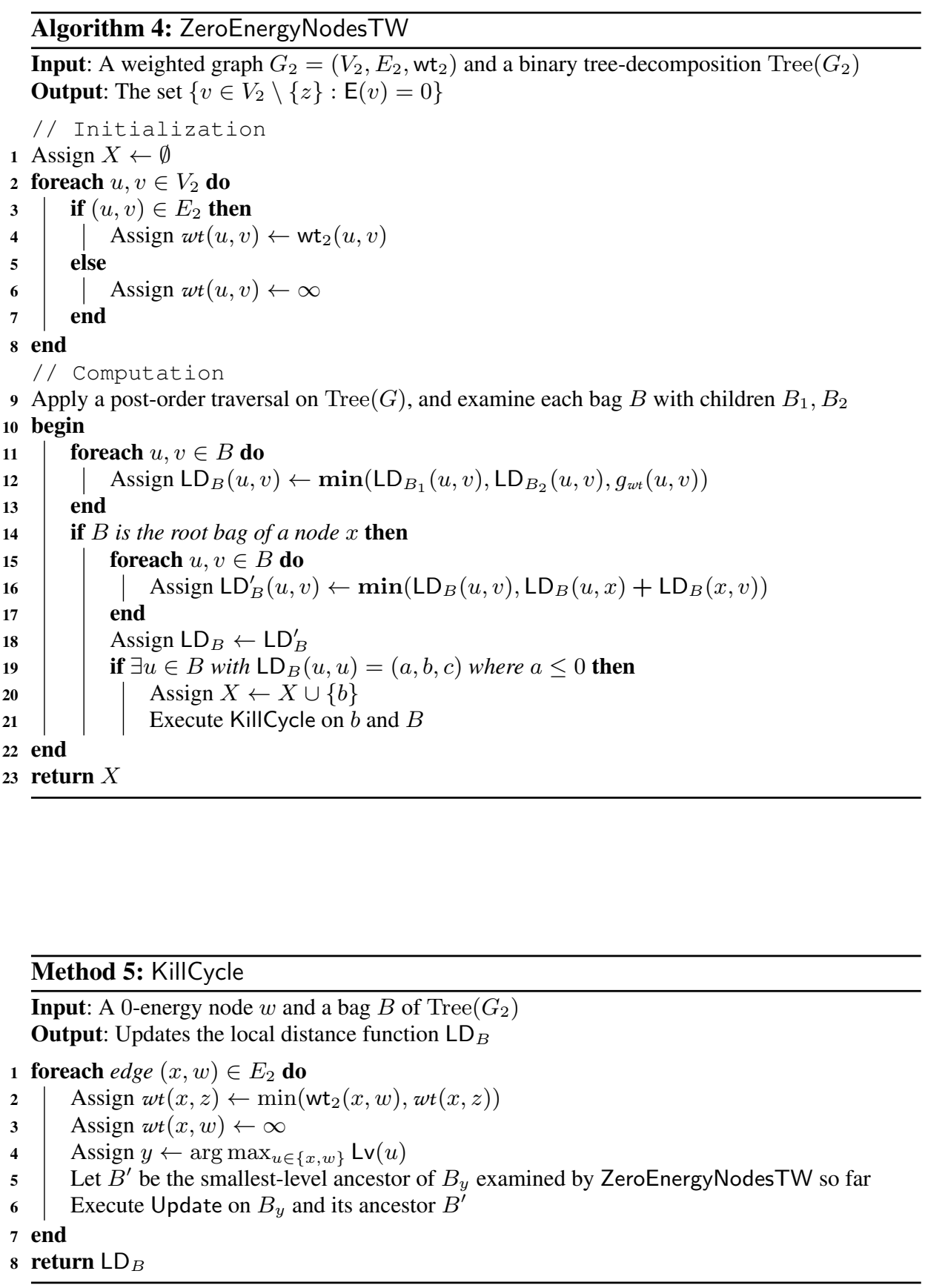


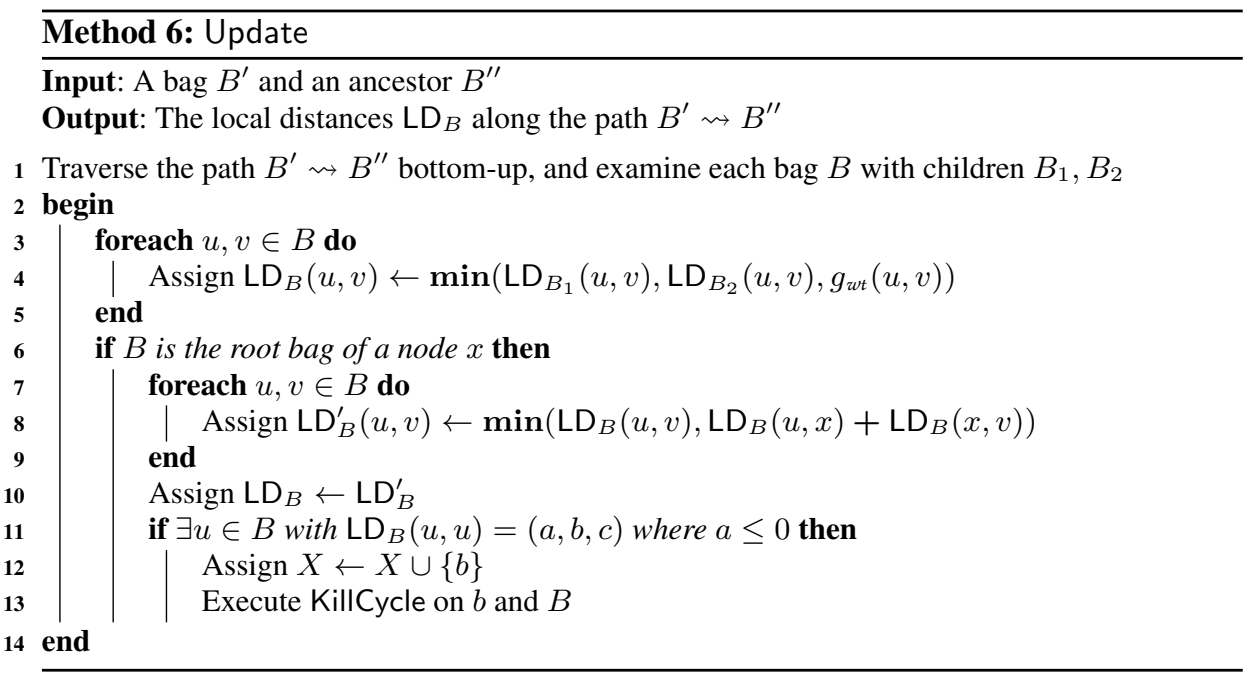

examines a bag $B$ (either directly, or through Update), every $\mathrm{U}$-shaped simple cycle in $B$ has been considered by the algorithm. This is true if no calls to KillCycle are made (if block in line 19), as then ZeroEnergyNodesTW is the same as MinCycle, and hence it follows from Lemma2

Now consider that KillCycle is called and $B^{\prime}$ is the smallest-level bag examined by ZeroEnergyNodesTW so far. Let $w$ be the 0 -energy node, $x$ an incoming neighbor of $w$, and $y=\arg \max _{u \in\{x, w\}} \operatorname{Lv}(u)$ (as in line 4 of KillCycle). By the definition of Ushaped paths, the edge $(x, w)$ appears only in paths that are $\mathrm{U}$-shaped in bags along the path $B_{y} \rightsquigarrow B^{\prime}$. Hence, after setting $w t(x, w)=\infty$ (line 3 of KillCycle), it suffices to update the local distance maps of these bags. Similarly, after setting wt $(x, z) \leftarrow$ $\min \left(\mathrm{wt}_{2}(x, w), w t(x, z)\right)$ (line 2 of KillCycle), since $B_{z}$ is the root of Tree $\left(G_{2}\right)$, it suffices to update the local distance maps in the bags along the path $B_{x} \rightsquigarrow B^{\prime}$. Either $x=y$, or, by the properties of tree-decompositions, $B_{x}$ is an ancestor of $B_{y}$. Hence in either case $B_{x} \rightsquigarrow B^{\prime}$ is a subpath of $B_{y} \rightsquigarrow B^{\prime}$, and both edge modifications in lines 2 and 3 are handled correctly by calling Update on $B_{y}$ and its ancestor $B^{\prime}$. The result follows.

Lemma 14. Algorithm ZeroEnergyNodesTW runs in $O(n \cdot \log n)$ time and $O(n)$ space.

Proof. Let $h=O(\log n)$ be the height of $\operatorname{Tree}\left(G_{2}\right)$.

1. The method Update performs a constant number of operations to each bag in the path $B^{\prime} \rightsquigarrow B^{\prime \prime}$ where $B^{\prime \prime}$ is ancestor of $B^{\prime}$, hence each call to Update requires $O(h)$ time.

2. The method KillCycle performs a constant number of operations locally and one call to Update for each incoming edge of $w$. Hence if $w$ has $k_{w}$ incoming edges, KillCycle requires $O\left(h \cdot k_{w}\right)$ time. Since KillCycle sets $w t(x, w)=\infty$ for all incoming edges of $w$, the node $w$ will not appear in non-positive cycles thereafter.

3. The algorithm ZeroEnergyNodesTW is similar to MinCycle which runs in $O(n)$ time and space (Lemma 5). The difference is in the additional if block in line 19 
Since KillCycle is called when a non-positive cycle is detected, it will be called at most once for each node $u \in V_{2} \backslash\{z\}$ (from either ZeroEnergyNodesTW or Update). It follows that the total time of ZeroEnergyNodesTW is

$$
O\left(n+\sum_{u}\left(h \cdot k_{u}\right)\right)=O\left(n+h \cdot\left|E_{2}\right|\right)=O(n \cdot \log n)
$$

where $k_{u}$ is the number of incoming edges of node $u$. Since KillCycle stores constant size of information in each bag of Tree $\left(G_{2}\right)$, the $O(n)$ space bound follows.

After the set $X$ of 0 -energy nodes has been computed, it remains to execute one instance of the single-source shortest path problem on the graph $\mathcal{G}^{|X|}$ (similarly as for our solution on general graphs). It is known that single-source distances in tree-decompositions of constant treewidth can be computed in $O(n)$ time [23|18]. We thus obtain the following theorem.

Theorem 7. Let $G=(V, E, \mathrm{wt})$ be a weighted graph of $n$ nodes with constant treewidth. The minimum initial credit value problem for $G$ can be solved in $O(n \cdot \log n)$ time and $O(n)$ space.

\section{Experimental Results}

\subsection{Minimum mean cycle}

We have implemented our approximation algorithm for the minimum mean cycle problem, and we let the algorithm run for as many iterations until a minimum mean cycle was discovered, instead of terminating after $O(\log (n / \epsilon))$ iterations required by Theorem 4 . We have tested its performance in running time and space against six other minimum mean cycle algorithms from Table 3 in control-flow graphs of programs. The algorithms of Burns and Lawler solve the more general ratio cycle problem, and have been adapted to the mean cycle problem as in [26].

\begin{tabular}{|c||c|c|c|c|c|c|}
\hline & Madani [38 & Burns [12] & Lawler [37] & Dasdan-Gupta [25] & Hartmann-Orlin [32 & Karp [35] \\
\hline \hline Time & $O\left(n^{2}\right)$ & $O\left(n^{3}\right)$ & $O\left(n^{2} \cdot \log (n \cdot W)\right)$ & $O\left(n^{2}\right)$ & $O\left(n^{2}\right)$ & $O\left(n^{2}\right)$ \\
\hline Space & $O(n)$ & $O(n)$ & $O(n)$ & $O\left(n^{2}\right)$ & $O\left(n^{2}\right)$ & $O\left(n^{2}\right)$ \\
\hline
\end{tabular}

Table 3: Asymptotic complexity of compared minimum mean cycle algorithms.

Setup. The algorithms were executed on control-flow graphs of programs from the DaCapo benchmark suit [3], obtained using the Soot framework [46]. For each benchmark we focused on graphs of at least 500 nodes. This supplied a set of medium sized graphs (between 500 and 1300 nodes), in which integer weights were assigned uniformly at random in the range $\left\{-10^{3}, \ldots, 10^{3}\right\}$. Memory usage was measured with [11].

Results. Figure 5 shows the average time and space performance of the examined algorithms (bars that exceeded the maximum value in the y-axis have been truncated). 
Our algorithm has much smaller running time than each other algorithm, in almost all cases. In terms of space, our algorithm significantly outperforms all others, except for the algorithms of Lawler, Burns, and Madani. Both ours and these three algorithms have linear space complexity, but ours also suffers some constant factor overhead from the tree-decomposition (i.e., the same node generally appears in multiple bags). Note that the strong performance of these three algorithms in space is followed by poor performance in running time.

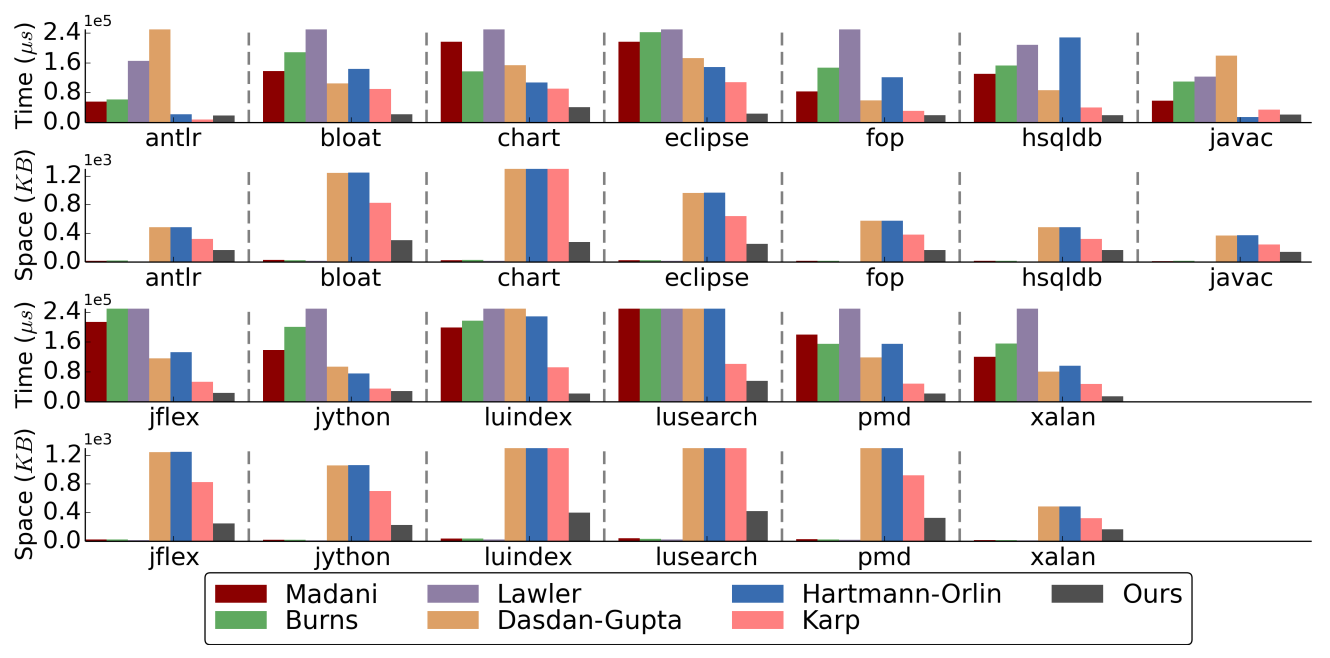

Fig. 5: Average performance of minimum mean cycle algorithms.

\begin{tabular}{|c||c|c|c|c|c|c|c|}
\hline & Madani & Burns & Lawler & Dasdan-Gupta & Hartmann-Orlin & Karp & Ours \\
\hline \hline antlr & 55814 & 61571 & 165789 & 284996 & 21893 & 7824 & $\mathbf{1 8 4 0 2}$ \\
\hline bloat & 138416 & 188356 & 350302 & 105145 & 144171 & 89949 & $\mathbf{2 2 3 9 1}$ \\
\hline chart & 216962 & 137112 & 573767 & 154062 & 107229 & 90717 & $\mathbf{4 0 8 9 0}$ \\
\hline eclipse & 216859 & 242323 & 667869 & 172792 & 148523 & 107864 & $\mathbf{2 3 4 8 6}$ \\
\hline fop & 83080 & 147384 & 406371 & 59176 & 121742 & 31557 & $\mathbf{1 9 3 0 6}$ \\
\hline hsqldb & 131041 & 153232 & 208328 & 86840 & 228632 & 40486 & $\mathbf{1 9 9 5 7}$ \\
\hline javac & 58443 & 110149 & 122996 & 179647 & 14719 & 34188 & $\mathbf{2 0 8 7 4}$ \\
\hline jllex & 214297 & 524822 & 554093 & 116820 & 133323 & 53329 & $\mathbf{2 3 8 6 0}$ \\
\hline jython & 139106 & 200922 & 503766 & 94052 & 75569 & 34864 & $\mathbf{2 8 7 6 0}$ \\
\hline luindex & 199650 & 217980 & 1240411 & 274319 & 228856 & 92379 & $\mathbf{2 2 1 4 2}$ \\
\hline lusearch & 433211 & 447280 & 1180051 & 263467 & 333297 & 101584 & $\mathbf{5 5 6 5 2}$ \\
\hline pmd & 180551 & 155118 & 585315 & 118578 & 155682 & 48326 & $\mathbf{2 1 9 7 8}$ \\
\hline xalan & 120897 & 156111 & 394458 & 81103 & 96873 & 47996 & $\mathbf{1 4 4 9 3}$ \\
\hline
\end{tabular}

Table 4: The time performance of Figure 5 (in $\mu s)$. 


\begin{tabular}{|c||c|c|c|c|c|c|c|}
\hline & Madani & Burns & Lawler & Dasdan-Gupta & Hartmann-Orlin & Karp & Ours \\
\hline \hline antlr & 16805 & 21018 & 11144 & 486435 & 489176 & 322384 & $\mathbf{1 6 8 6 4 8}$ \\
\hline bloat & 29723 & 24500 & 19458 & 1245272 & 1249444 & 826645 & $\mathbf{3 0 6 0 2 6}$ \\
\hline chart & 27130 & 30567 & 18172 & 2025448 & 2029294 & 1347048 & $\mathbf{2 7 8 5 8 6}$ \\
\hline eclipse & 24215 & 26488 & 16293 & 965063 & 968595 & 640720 & $\mathbf{2 5 4 3 9 3}$ \\
\hline fop & 16845 & 17975 & 11052 & 576174 & 578646 & 382338 & $\mathbf{1 6 9 7 3 8}$ \\
\hline hsqldb & 16798 & 19309 & 11144 & 486435 & 489096 & 322384 & $\mathbf{1 6 8 6 4 8}$ \\
\hline javac & 14681 & 17047 & 9664 & 372697 & 375453 & 247019 & $\mathbf{1 4 4 7 2 1}$ \\
\hline jflex & 24561 & 26946 & 16322 & 1244495 & 1248036 & 826743 & $\mathbf{2 5 1 5 4 9}$ \\
\hline jython & 22518 & 23337 & 14899 & 1059291 & 1062570 & 703581 & $\mathbf{2 2 8 2 0 7}$ \\
\hline luindex & 39309 & 40223 & 25604 & 3521607 & 3526792 & 2342833 & $\mathbf{3 9 9 0 7 6}$ \\
\hline lusearch & 41488 & 33350 & 26991 & 3387914 & 3393343 & 2253403 & $\mathbf{4 2 2 6 7 9}$ \\
\hline pmd & 32204 & 24481 & 21021 & 1391551 & 1395786 & 923975 & $\mathbf{3 2 6 1 3 7}$ \\
\hline xalan & 16798 & 17763 & 11144 & 486435 & 489102 & 322384 & $\mathbf{1 6 8 6 4 8}$ \\
\hline
\end{tabular}

Table 5: The space performance of Figure 5 (in KB).

\subsection{Minimum initial credit}

We have implemented our algorithm for the minimum initial credit problem on general graphs and experimentally evaluated its performance on a subset of benchmark weighted graphs from the DIMACS implementation challenges [1]. Our algorithm was tested against the existing method of [9]. The direct implementation of the algorithm of [9] performed poorly, and for this we also implemented an optimized version (using techniques such as caching of intermediate results and early loop termination).

Setup. For each input graph we first computed its minimum mean value $\mu^{*}$ using Karp's algorithm, and then subtracted $\mu^{*}$ from the weight of each edge to ensure that at least one non-positive cycle exists (thus the energies are finite).

Results. Figure 6] depicts the running time of the algorithm of [9] (with and without optimizations) vs our algorithm. A timeout was forced at $10^{10} \mu \mathrm{s}$. Our algorithm is orders of magnitude faster, and scales better than the existing method.

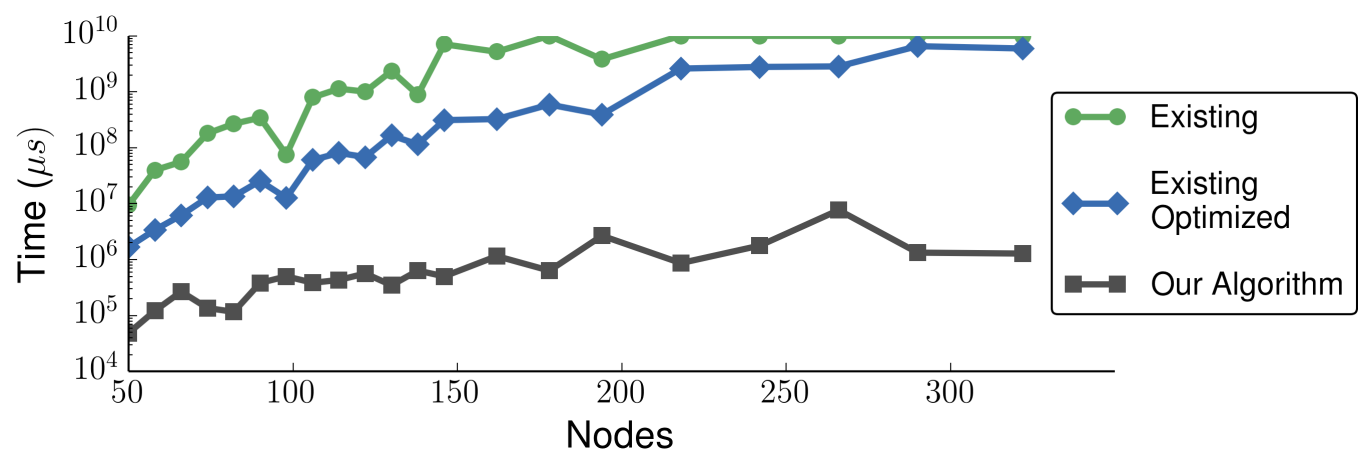

Fig. 6: Comparison of running times for the minimum initial credit problem. 


\begin{tabular}{|c||c|c|c|}
\hline$n$ & Existing & Existing Optimized & Ours \\
\hline \hline 50 & 9453565 & 1680924 & $\mathbf{4 8 6 3 5}$ \\
\hline 58 & 39744129 & 3394193 & $\mathbf{1 2 1 7 7 4}$ \\
\hline 66 & 55766874 & 6201044 & $\mathbf{2 6 7 8 2 5}$ \\
\hline 74 & 180080064 & 12833610 & $\mathbf{1 3 6 2 3 9}$ \\
\hline 82 & 267993314 & 13563936 & $\mathbf{1 1 6 5 1 8}$ \\
\hline 90 & 342779026 & 25453589 & $\mathbf{3 8 3 2 9 2}$ \\
\hline 98 & 74622910 & 12648395 & $\mathbf{5 0 1 3 6 5}$ \\
\hline 106 & 791441986 & 60294150 & $\mathbf{3 8 5 7 9 9}$ \\
\hline 114 & 1133055323 & 80584700 & $\mathbf{4 3 2 2 9 0}$ \\
\hline 122 & 1004898322 & 67982455 & $\mathbf{5 6 4 8 3 8}$ \\
\hline 130 & 2354354250 & 165193753 & $\mathbf{3 4 8 1 1 2}$ \\
\hline 138 & 881117317 & 114743182 & $\mathbf{6 3 6 4 8 1}$ \\
\hline 146 & 7050113907 & 311146051 & $\mathbf{5 0 1 3 1 4}$ \\
\hline 162 & 5179877563 & 324877384 & $\mathbf{1 1 5 4 4 4 7}$ \\
\hline 178 & Timeout & 589873640 & $\mathbf{6 3 5 1 5 5}$ \\
\hline 194 & 3799301931 & 391240954 & $\mathbf{2 6 7 2 1 2 7}$ \\
\hline 218 & Timeout & 2596083382 & $\mathbf{8 6 6 2 1 3}$ \\
\hline 242 & Timeout & 2774469734 & $\mathbf{1 7 7 9 5 1 2}$ \\
\hline 266 & Timeout & 2839496222 & $\mathbf{7 6 7 6 6 3 8}$ \\
\hline 290 & Timeout & 6526762301 & $\mathbf{1 3 3 2 4 0 3}$ \\
\hline 322 & Timeout & 5929433611 & $\mathbf{1 2 8 2 2 5 8}$ \\
\hline
\end{tabular}

Table 6: The time performance of Figure 6 in $\mu s$. 


\section{References}

1. DIMACS implementation challenges, http://dimacs.rutgers.edu/ Challenges/

2. Almagor, S., Boker, U., Kupferman, O.: Formalizing and reasoning about quality. In: ICALP. pp. 15-27 (2013)

3. Blackburn, S.M.e.a.: The dacapo benchmarks: Java benchmarking development and analysis. In: OOPSLA (2006)

4. Bloem, R., Chatterjee, K., Henzinger, T.A., Jobstmann, B.: Better quality in synthesis through quantitative objectives (2015)

5. Bloem, R., Greimel, K., Henzinger, T.A., Jobstmann, B.: Synthesizing robust systems. In: FMCAD. pp. 85-92 (2009)

6. Bodlaender, H.L.: A tourist guide through treewidth. Acta Cybern. (1993)

7. Bodlaender, H.: Discovering treewidth. In: SOFSEM 2005: Theory and Practice of Computer Science, LNCS, vol. 3381. Springer (2005)

8. Boker, U., Chatterjee, K., Henzinger, T.A., Kupferman, O.: Temporal specifications with accumulative values. In: LICS. pp. 43-52 (2011)

9. Bouyer, P., Fahrenberg, U., Larsen, K.G., Markey, N., Srba, J.: Infinite runs in weighted timed automata with energy constraints. In: Formal Modeling and Analysis of Timed Systems, Lecture Notes in Computer Science, vol. 5215, pp. 33-47. Springer Berlin Heidelberg (2008)

10. Bouyer, P., Markey, N., Matteplackel, R.M.: Averaging in LTL. In: CONCUR. pp. 266-280 (2014)

11. Brosius, D.: Java agent for memory measurements, https://github.com/jbellis/ jamm

12. Burns, S.M.: Performance analysis and optimization of asynchronous circuits. Tech. rep. (1991)

13. Cerny, P., Henzinger, T.A., Radhakrishna, A.: Quantitative abstraction refinement. In: POPL. pp. 115-128 (2013)

14. Chatterjee, K., Lacki, J.: Faster algorithms for Markov decision processes with low treewidth. In: CAV (2013)

15. Chatterjee, K., Doyen, L., Edelsbrunner, H., Henzinger, T.A., Rannou, P.: Mean-payoff automaton expressions. CoRR abs/1006.1492 (2010)

16. Chatterjee, K., Doyen, L., Henzinger, T.A.: Expressiveness and closure properties for quantitative languages. LMCS 6(3) (2010)

17. Chatterjee, K., Doyen, L., Henzinger, T.A.: Quantitative languages. ACM Trans. Comput. Log. 11(4) (2010)

18. Chatterjee, K., Goyal, P., Ibsen-Jensen, R., Pavlogiannis, A.: Faster algorithms for algebraic path properties in recursive state machines with constant treewidth. In: POPL (2015)

19. Chatterjee, K., Henzinger, M., Krinninger, S., Loitzenbauer, V., Raskin, M.A.: Approximating the minimum cycle mean. Theor. Comput. Sci. (2014)

20. Chatterjee, K., Henzinger, T.A., Jobstmann, B., Singh, R.: Measuring and synthesizing systems in probabilistic environments. In: JACM. pp. 380-395 (2015)

21. Chatterjee, K., Henzinger, T.A., Otop, J.: Nested weighted automata. Tech. rep., IST Austria (2014)

22. Chatterjee, K., Velner, Y.: Mean-payoff pushdown games. In: LICS (2012)

23. Chaudhuri, S., Zaroliagis, C.D.: Shortest Paths in Digraphs of Small Treewidth. Part I: Sequential Algorithms. Algorithmica (1995)

24. Courcelle, B.: The monadic second-order logic of graphs. i. recognizable sets of finite graphs. Information and Computation 85

25. Dasdan, A., Gupta, R.: Faster maximum and minimum mean cycle algorithms for systemperformance analysis. Computer-Aided Design of Integrated Circuits and Systems, IEEE Transactions on 17(10), 889-899 (Oct 1998) 
26. Dasdan, A., Irani, S.S., Gupta, R.K.: An experimental study of minimum mean cycle algorithms. Tech. rep. (1998)

27. Droste, M., Kuich, W., Vogler, H.: Handbook of Weighted Automata. Springer Publishing Company, Incorporated, 1st edn. (2009)

28. Droste, M., Meinecke, I.: Weighted automata and weighted mso logics for average and longtime behaviors. Inf. Comput. 220, 44-59 (2012)

29. Elberfeld, M., Jakoby, A., Tantau, T.: Logspace versions of the theorems of Bodlaender and Courcelle. In: FOCS (2010)

30. Gustedt, J., Mhle, O., Telle, J.: The treewidth of java programs. In: Algorithm Engineering and Experiments. LNCS, Springer (2002)

31. Halin, R.: S-functions for graphs. Journal of Geometry (1976)

32. Hartmann, M., Orlin, J.B.: Finding minimum cost to time ratio cycles with small integral transit times. NETWORKS 23, 567-574 (1993)

33. Henzinger, T.A., Otop, J.: From model checking to model measuring. In: CONCUR. pp. 273-287 (2013)

34. Karp, R.M.: A characterization of the minimum cycle mean in a digraph. Discrete Mathematics 23(3), 309 - 311 (1978)

35. Karp, R.M.: A characterization of the minimum cycle mean in a digraph. Discrete Mathematics (1978)

36. Kwek, S., Mehlhorn, K.: Optimal search for rationals. Inf. Process. Lett. 86(1), 23-26 (2003)

37. Lawler, E.: Combinatorial Optimization: Networks and Matroids. Saunders College Publishing (1976)

38. Madani, O.: Polynomial value iteration algorithms for deterministic MDPs. In: UAI'02 (2002)

39. Obdrzálek, J.: Fast mu-calculus model checking when tree-width is bounded. In: CAV (2003)

40. Orlin, J.B., Ahuja, R.K.: New scaling algorithms for the assignment and minimum mean cycle problems. Math. Program. (1992)

41. Papadimitriou, C.H.: Efficient search for rationals. Information Processing Letters 8(1), 1 4 (1979)

42. Reed, B.A.: Finding approximate separators and computing tree width quickly. In: STOC (1992)

43. Robertson, N., Seymour, P.: Graph minors. iii. planar tree-width. Journal of Combinatorial Theory, Series B (1984)

44. Tarjan, R.: Depth-first search and linear graph algorithms. SIAM Journal on Computing (1972)

45. Thorup, M.: All Structured Programs Have Small Tree Width and Good Register Allocation. Information and Computation (1998)

46. Vallée-Rai, R., Co, P., Gagnon, E., Hendren, L., Lam, P., Sundaresan, V.: Soot - a java bytecode optimization framework. In: CASCON' 99. IBM Press (1999)

47. Velner, Y.: The complexity of mean-payoff automaton expression. In: ICALP (2012) 